\title{
Transgenerational inheritance of BPA-induced obesity correlates with transmission of new CTCF sites in the Fto gene
}

\author{
Yoon Hee Jung ${ }^{1}$, Brianna J. Bixler ${ }^{1}$, Daniel Ruiz', Hsiao-Lin V. Wang ${ }^{1}$, Hannah Linsenbaum ${ }^{1}$, \\ Jian-Feng Xiang ${ }^{1}$, Andrew M. Shafik ${ }^{1}$, Peng Jin ${ }^{1}$, and Victor G. Corces ${ }^{1}$ \\ 1'Department of Human Genetics, Emory University School of Medicine, 615 Michael St, Atlanta, \\ GA 30322, USA
}

Running Title: Transgenerational epigenetics

Key words: Chromatin; Transcription; Enhancer; Sperm; Oocyte; Fertilization; 3D organization

Corresponding author: Victor G. Corces, Department of Human Genetics, Emory University School of Medicine, 615 Michael Street, Atlanta, GA 30322. Phone number +1 4047275947. Email vgcorces@gmail.com 


\begin{abstract}
The mechanisms by which epiphenotypes are transmitted transgenerationally through the parental germlines are poorly understood. Here we show that exposure of pregnant mouse F0 females during E7.5-E13.5 to bisphenol A results in obesity in the F2 progeny in the absence of additional exposure. This epiphenotype can be transmitted through the male and female germlines up to the F5 generation, decreases in F6, and disappears in F7. Analysis of chromatin changes in the sperm of the $F 1$ generation reveals a widespread increase in chromatin accessibility at binding sites for CTCF and other transcription factors accompanied by alterations in 3D organization. Comparison of the transmission of obesity between F2 and F5 and its disappearance in F7 with alterations in the binding of these transcription factors points to the activation of an enhancer in an intron of the Fto gene as the cause of transgenerational inheritance. Activation of the Fto enhancer results in a decrease of $\mathrm{m}^{6} \mathrm{~A}$ in sperm RNAs, which may result in alterations of gene expression in the embryo after fertilization. Given the established involvement of SNPs in FTO in human obesity, the results suggest that both genetic and epigenetic alterations of the same gene can lead to the same phenotypic outcomes on human health.
\end{abstract}

\title{
Main text
}

Evidence suggestive of inter and transgenerational inheritance of epiphenotypes in mammals has been reported extensively. Numerous studies suggest a link between parental environments and a variety of effects in the offspring (1-3). For example, exposure of laboratory animals or humans to endocrine disrupting compounds leads to increased reproductive dysfunction, cancer, obesity, diabetes, and behavioral disorders (4). However, we still lack an understanding of the molecular processes by which alterations induced by environmental exposures are transmitted between the exposed and subsequent generations. This is in part due to the peculiar cellular characteristics of mammalian sperm, which contain a highly compact nucleus relative to normal somatic cells and, until recently, have been thought to contain little transmissible epigenetic information. With the exception of some classes of transposable elements, DNA methylation is erased twice in every generation - during germ cell development and after fertilization - making this modification, on its own, a poor candidate to carry epigenetic information between generations (5). Mouse and human sperm retain $8-15 \%$ of the histones present in somatic cells, and these histones contain covalent modifications characteristic of active and silenced chromatin at specific genes (6-10). Many of these histone modifications are erased after fertilization when protamines are replaced by histones in the zygote, bringing into question whether histone modifications can carry epigenetic information between generations (11). Small RNAs, including miRNAs and tRNA fragments, present in sperm are transferred to the zygote and may cause changes in transcription affecting the differentiation of adult tissues (12-15). However, it is difficult to rationalize how this information can be passed to subsequent generations beyond F1 to explain transgenerational phenomena. It is possible that transgenerational inheritance of epiphenotypes involves more than one of these candidates. For example, the interplay between placeholder nucleosomes containing the histone variant $\mathrm{H} 2 \mathrm{~A} . \mathrm{Z}$ and DNA methylation has been shown to mediate transmission of information between the gametes and embryos in zebrafish (16). In this context, recent results indicate that mammalian sperm chromatin contain a variety of transcription factors (TFs). The interaction of these TFs with DNA can affect DNA methylation during the reprograming of the germline and preimplantation development (17). If these interactions are affected by the parental environment, they could be transmitted to subsequent generations in an altered state to elicit phenotypic changes in the progeny (11).

\section{Exposure of pregnant females to BPA causes obesity that can be transmitted transgenerationally}


To gain insights into the mechanisms by which epiphenotypes elicited by environmental exposures can be transmitted transgenerationally, we examined the effect of the endocrine disrupting chemical Bisphenol A (BPA). A large body of work by Skinner and collaborators has shown that exposure of pregnant rats and mice to a variety of endocrine disruptors during the window of time when the germline of the embryo is demethylated leads to various health effects in the progeny, including testicular and breast cancer, behavioral defects, and obesity $(18,19)$. Following on this pioneering work, we exposed pregnant mouse females (F0) via daily intraperitoneal injection of BPA (50 mg/kg) during days E7.5 through E13.5 of fetal development (20). As a control, pregnant females were injected with vehicle only. In the rest of the manuscript we will refer to the progeny of BPA-exposed Fo females as BPA-Fi, where $i$ is the generation number, and the progeny of vehicle-exposed females as CTL-Fi, although the exposure took place only in the F0 generation. The BPA-F1 progeny has no overt phenotypic abnormalities when compared to CTL-F1 and their body weight is the same as that of controls (Fig. 1A). BPA-F1 males were then crossed to BPA-F1 females from a parallel exposure experiment (Fig. S1A). BPA-F2 males and females displayed an obvious increase in body weight with respect to CTL-F2 animals. These crosses were continued, and the increased body weight was also observed in the BPA-F3 through BPA-F6 generations, but it was completely lost in BPA-F7 animals (Fig. 1A). To compare changes in body weight in different generations, we took the median weight of control males at week 10 and we defined obese animals as those whose weight is one standard deviation above the median control weight. Only $5 \%$ of males are overweight in the BPA-F1 generation by this criterion, which is similar to control (Fig. 1B). The fraction of overweight males increases to $65 \%$ in BPA-F2, peaks at $97 \%$ in BPA-F4, and starts decreasing through BPA-F4-6. All BPA-F7 males display weights similar to those of control males (Fig. 1B). Body weight of BPA-Fi animals is the same as that of controls at 3 weeks of age, but differences become statistically significant by weeks 5-6 and remain for the rest of adult life (Fig. 1C). These differences are more pronounced in males than females (Fig. 1C and S1B). Crosses between overweight and between lean BPA-F6 animals produce progeny with weights statistically indistinguishable from those of controls (Fig. S1C), suggesting that the germline of F6 animals has lost the information responsible for the inheritance of obesity independent of the weight of the animals. These experiments were performed 6 different times with similar results. BPA-Fi mice show a large accumulation of visceral white adipose tissue (WAT) compared to controls (Fig. 1D, top), and increased number of adipocytes and accumulation of lipid droplets (Fig. 1D, bottom). The median weight for CTL-F4 males is $39.2 \mathrm{~g}$ and for BPA-F4 males is $51.1 \mathrm{~g}$ (Fig. S1D). Whereas the visceral WAT represents $1.32 \%$ of total body weight in control male animals, this value increases to $2.45 \%$ in the F4 male progeny of F0 females exposed to BPA (Fig. S1E). To understand the origin of the higher number of adipocytes present in BPA-F4 fat tissue, we examined its composition in more detail by performing immunofluorescence microscopy with antibodies to Sca1 , which specifically labels adipocyte progenitor cells (Fig. S1F). Results show that $69 \%$ of the cells in fat tissue from BPA-F4 animals correspond to adipocyte progenitors compared to $22 \%$ in the fat tissue of control animals (Fig. S1G).

It has been estimated that humans are exposed to an average of $0.05 \mathrm{mg} / \mathrm{kg}$ body weight of BPA or less (21), resulting in concentrations of this compound in serum in the ng per $\mathrm{ml}$ range (22). Since the amount of BPA to which mouse pregnant females are exposed is much higher than that to which the average human is thought to be exposed, we injected pregnant females with decreasing concentrations of BPA and examined the effect on body weight in the progeny. The results indicate that BPA at concentrations of $50 \mu \mathrm{g} / \mathrm{kg}$, which are comparable to those to which humans are normally exposed, cause a similar increase in body weight as higher concentrations (Figure $\mathrm{S} 1 \mathrm{H}$ ). To determine the amount of BPA in the exposed embryo compared to that injected in pregnant females, we measured BPA levels using mass spectroscopy at 

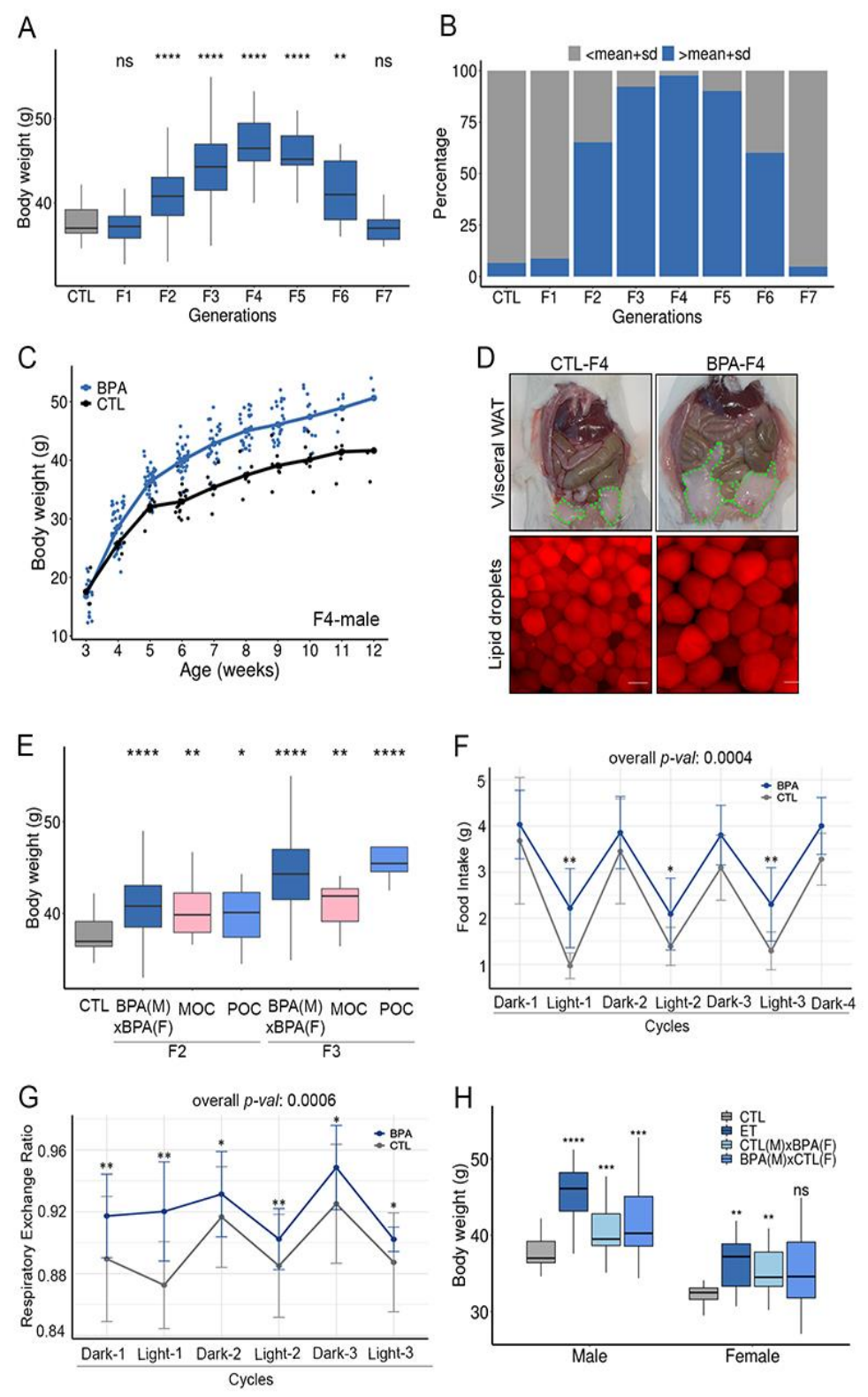

Fig. 1. Analysis of BPA-induced obesity.

(A) Changes in body weight between control and different generations of the progeny of BPA-exposed F0 mouse females. (B) Percentage of overweight mice in the F1-F7 generation progeny of F0 BPA-exposed females. (C) Changes in body weight with time in control and the F4 generation of progeny from BPA-exposed females. (D) Differences in visceral fat, stored lipids, and adipocyte size in BPA-F4 versus control; bar represents $50 \mu \mathrm{m}$. (E) Body weight difference in males between control and the progeny from maternal or paternal outcrosses between control and BPA-F2 or BPA-F3. (F) Food intake by BPA-F4 and CTL-F4 males during a 3-day period. (G) Differences in respiratory exchange ratio between BPA-F4 and CTL-F4 males over a 3-day period. $(\mathbf{H})$ Body weight difference in males and females between control, progeny from embryo transfer (ET) experiments, and outcrosses between BPA-F2 and control. For all panels, $P$ values were calculated using t-test with respect to control; ${ }^{* * * *} p<0.0001,{ }^{* * *}<0.001,{ }^{* *}$ $p<0.01$. 
different times after injection. We found that the amount of BPA in embryonic tissues is $1.5 \mathrm{mg} / \mathrm{kg}$ $10 \mathrm{~min}$ after injection, rapidly decreases by 500 -fold to $0.105 \mathrm{mg} / \mathrm{kg}$ after $120 \mathrm{~min}$ and is undetectable by $24 \mathrm{~h}$. This suggests that BPA is rapidly eliminated or metabolized and that a very transient daily exposure is able to elicit the effects described here.

\section{Obesity can be transmitted through the paternal or maternal germlines}

In all experiments described so far, obesity was observed in the F2 progeny from two F1 mice from two parallel different exposures (Figure S1A). Therefore, BPA could have affected either of the two parental germlines or both. To determine whether the obese epiphenotype can be inherited through the paternal or maternal germlines, we outcrossed the F1 progeny to control unexposed mice of the opposite sex (Figure S2A). We then measured the body weight of males from the F2 and F3 generations. We find that the median weight increases in the F2 progeny and raises further in the F3 independent of the parental origin of the exposed germline, suggesting that the obese epiphenotype can be inherited through both the paternal and maternal germlines (Figure 1E).

To gain insights into the physiological processes underlying the obesity phenotype, we housed mice in metabolic cages and measured food intake and energy use. BPA-F4 mice show an increase in total food consumption with respect to controls (Fig. S2B). This increase is due to additional eating during the fasting light cycles (Fig. 1F and S2D). In agreement with this observation, the respiratory exchange ratio of BPA-F4 mice shows less of the characteristic active cycle peaks reflecting preferential carbohydrate utilization, and fasting cycle troughs indicating preferential fat utilization (23). Rather, a respiratory exchange ratio indicative of more constant carbohydrate preference was maintained throughout the 3-day metabolic cage test (Fig. 1G and $\mathrm{S} 2 \mathrm{E}$ ). Together, these results suggest that BPA-F4 mice exhibit unregulated eating patterns that reduce their ability to utilize fat as a fuel source, resulting in increased adiposity. Similar results were obtained by comparing overweight and lean BPA-F6 mice (Figure S2C). BPA-F6 lean mice i.e. those similar in weight to control, eat less during the light cycle than BPA-F6 overweight mice (Fig. S2F and S2G), and the respiratory exchange rate does not oscillate between day and night (Fig. S2H and S2I).'

To ensure that the obese phenotype is not the result of external factors such as the maternal environment, transfer of the maternal microbiome, presence of constituents other than sperm in the seminal fluid, etc., we performed in vitro fertilization using purified oocytes from a BPA-F2 female and sperm isolated from the cauda epididymis of a CTL-F2 male. The resulting embryos were then implanted into pseudo pregnant unexposed control females and the weight of their progeny was measured at 10 weeks of age. As controls, CTL-F2 males were directly crossed with BPA-F2 females and vice-versa. The results indicate that the median weight of males and females from the embryo transfer experiment is significantly higher than that of controls (Fig. $1 \mathrm{H})$, suggesting that the overweight phenotype is indeed due to epigenetic changes in the germline rather than external factors.

\section{Exposure to BPA results in alterations of CTCF occupancy and 3D interactions in the germline}

Since BPA is an estrogen-like compound that has been shown to act through a variety of nuclear hormone receptors (24), we hypothesized that exposure to BPA may cause alterations in transcription accompanied by a redistribution of TFs throughout the genome of the germline during E7.5-E13.5, the time of embryonic development when the germline DNA becomes demethylated. The unmethylated state of the DNA may allow binding of TFs to new sites in the genome where these TFs cannot bind when the DNA is methylated. Subsequently, the presence of bound TFs at these new sites may protect the DNA from re-methylation and these changes 


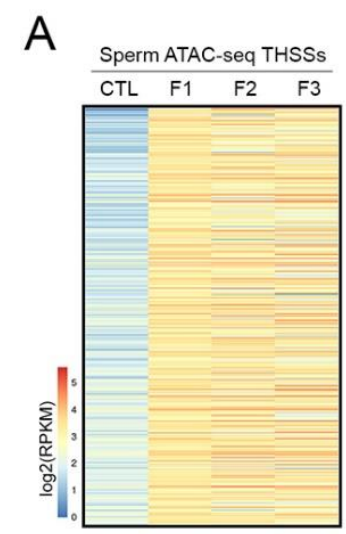

$\mathrm{B}$
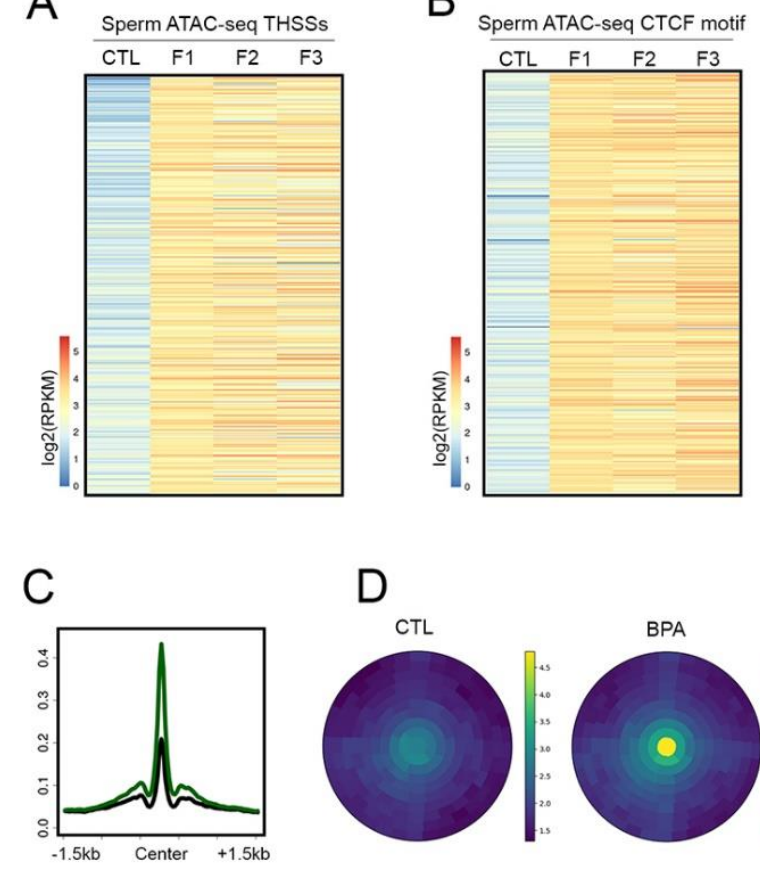
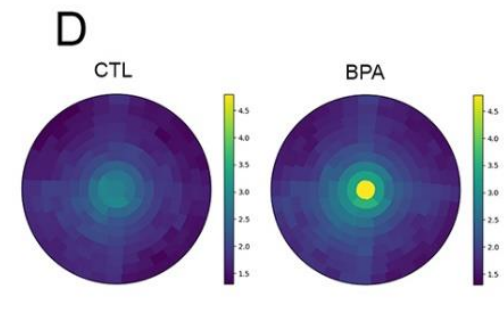

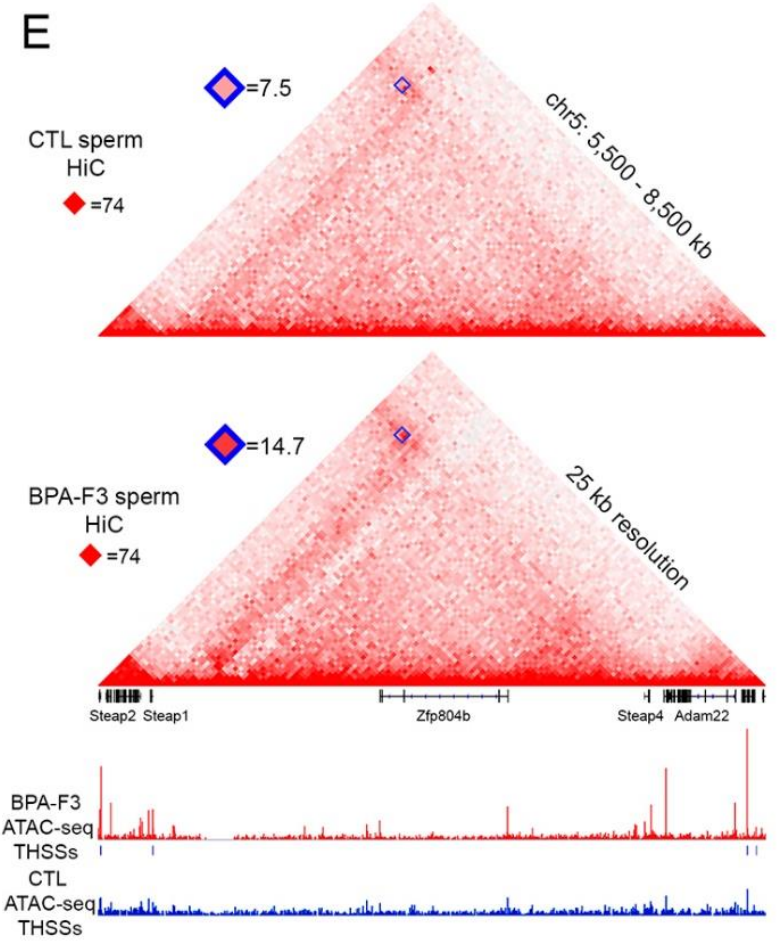

THSSs

Fig. 2. Effect of BPA on TF occupancy and 3D genome architecture.

(A) Heatmap of ATAC-seq sites differentially accessible in sperm between BPA-F1 and CTL-F1 and their changes in accessibility in sperm of BPA-F2-F3. (B) Heatmap of ATACseq sites containing CTCF motifs differentially accessible between sperm of BPA-F1 and CTL-F1 and their changes in accessibility in sperm of BPA-F2-F3. (C) Average ATAC-seq accessibility at site differentially accessible in sperm of BPA-F1 (green) and CTL-F1 (black). (D) Frequency of interactions mediated by CTCF sites differentially accessible in sperm of BPA-F1 versus CTL-F1; Hi-C data is from sperm of F3. (E) Specific example of a CTCF loop whose anchors interact more frequently in sperm of BPA-F3 than CTL-F3.

may persist in the mature germline (17). To test this possibility, we performed ATAC-seq using the OmniATAC protocol (25) in sperm isolated from the cauda epididymis of control and BPA F1F3 males. To analyze the effect of BPA on the germline of the F1 embryo present in the exposed F0 female, we first identified differences between BPA-F1 and CTL-F1 sperm using a cutoff of FDR 0.05 and $>2$-fold difference in sub-nucleosome-size ATAC-seq signal, which corresponds to the presence of bound TFs in chromatin (26). We will refer to these sites as Transposase Hypersensitive Sites (THSSs). We found 1,327 differential THSSs between the two samples (Fig. $2 A$ ), suggesting a dramatic redistribution of TFs in sperm as a consequence of exposure of germ line cells to BPA. The differential sites observed in BPA-F1 sperm correspond to a variety of TFs but, surprisingly, 107 sites contain the CTCF motif (Figure 2B and Fig. S3A). Some of these sites are maintained in sperm of the F2 and F3 generations whereas other sites disappear. In addition, new sites not present in the F1 germline can be observed in subsequent generations, suggesting that the effect of BPA can be altered after the initial exposure (Fig. 2A and 2B). The accessibility at $\mathrm{F} 1$ sites, which measures levels of protein present, is higher in sperm of BPA-F1 than control mice (Fig. 2C). A specific example of increased ATAC-seq signal at CTCF and androgen receptor (Ar) sites in sperm of BPA-F1 not present in control but maintained through F3 is shown in Fig. S3C. To test whether the new CTCF sites observed in sperm of BPA mice affect the 3D organization of sperm chromatin, we performed Hi-C (see Supplemental Table 1 for quality 
controls of the Hi-C libraries) in sperm of BPA-F3 and CTL-F3, and compared interactions between anchors containing differential CTCF sites and other CTCF sites in the genome using SIP (27) (Fig. S3B). Results indicate a dramatic increase in interactions observed in Hi-C heatmaps as punctate signal, indicating the formation of new CTCF loops (Fig. 2D). An example of changes in CTCF levels in BPA-F3 sperm and the formation of a loop between CTCF sites adjacent to Steap1 and Steap4, a gene shown to be involved in obesity in humans (28), is shown in Fig. 2E. These results suggest that BPA, directly or indirectly, induces the binding of CTCF and various TFs at different regions in the genome in the sperm of the F1 generation. Some of these changes are maintained in subsequent generations, altering the 3D organization of chromatin in mature sperm.

\section{Transcription factors with altered occupancy in the germline are inherited transgenerationally and mediate enhancer-promoter interactions}

The transgenerational transmission of obesity decreases substantially in the F6 generation, suggesting that epigenetic alterations induced by exposure to BPA start disappearing in the sperm of F5. Therefore, to uncover the alterations in TF binding responsible for transgenerational inheritance, we identified ATAC-seq THSSs present or absent in generations BPA-F1-F4 with respect to control using an 0.05 FDR and $\geq 2$-fold change cutoff and requiring that differences are consistently present or absent, respectively, in all replicates of each sample. Using these criteria, we identified 69 sites that are present in two different replicates of sperm from BPA F1-F4 males but not in controls, and 5 sites that are present in sperm of controls but not BPA animals (Fig. 3A and Fig. S4A). Visual inspection of these 5 sites suggests that they correspond to peaks with very low ATAC-seq signal in sperm of control males (Fig. S4B). Since their biological significance is unclear, they will not be considered further. Most of the 69 BPA-gained sites are present in distal intergenic regions or introns, suggesting that they may correspond to regulatory sequences such as enhancers (Fig. S4C). Motif analysis at the summits of ATAC-seq peaks at these sites indicates the presence of motifs for a variety of TFs such as CTCF, Znf143, Foxa1, and several nuclear hormone receptors, including Esr1/2, AR, and Ppary (Fig. 3B). Thirty-four of these sites contain binding motifs for CTCF plus another TF in very close proximity (Fig. S4D). To explore the relationship between the presence of these TFs at new sites in the sperm genome and possible changes in DNA methylation that could account for their differential binding to DNA, we performed genome wide BS-seq (GWBS) in sperm of control and BPA-F3 males. We find a large number of differentially methylated regions (DMRs) defined as a change of $>20 \%$ in methylation levels and a $p$ value $\leq 0.0001$ (Fig. S4E). Of these, 1,428 regions that are hypomethylated in sperm of BPAF3 with respect to control and 648 hypermethylated regions, suggesting that exposure of the germline to BPA induces DMRs that persist transgenerationally. These DMRs are located almost exclusively in distal intergenic and intronic regions, suggesting that they may correspond to enhancers (Fig S4F). We then explored the relationship between the observed changes in ATACseq signal between BPA and control versus the change in CpG methylation in a 300 bp region surrounding the summit of the 69 ATAC-seq peaks maintained in BPA-F1 through F4. Sites with increased accessibility in BPA sperm are generally hypomethylated with respect to control, especially those containing the binding motif for CTCF, whose interaction with DNA is known to be sensitive to methylation (Fig. $3 \mathrm{C}$ and Fig. S4D). These observations agree with the possibility that BPA-induced alterations of transcription in PGCs at the time when the DNA is being demethylated may allow binding of CTCF and other TFs to specific sites in the genome, which are then protected from re-methylation when this process occurs after E13.5 in the male germline. These sites may then become enhancers in an epigenetically active state in sperm. To explore this possibility, we called significant interactions in the $\mathrm{Hi}-\mathrm{C}$ data using Fit- $\mathrm{Hi}-\mathrm{C}$ and identified genes whose promoters are contacted by the 69 putative enhancers in BPA-F3 sperm. Results show that a total of 610 gene promoters are contacted by these 69 sites. Metaplots of interaction 

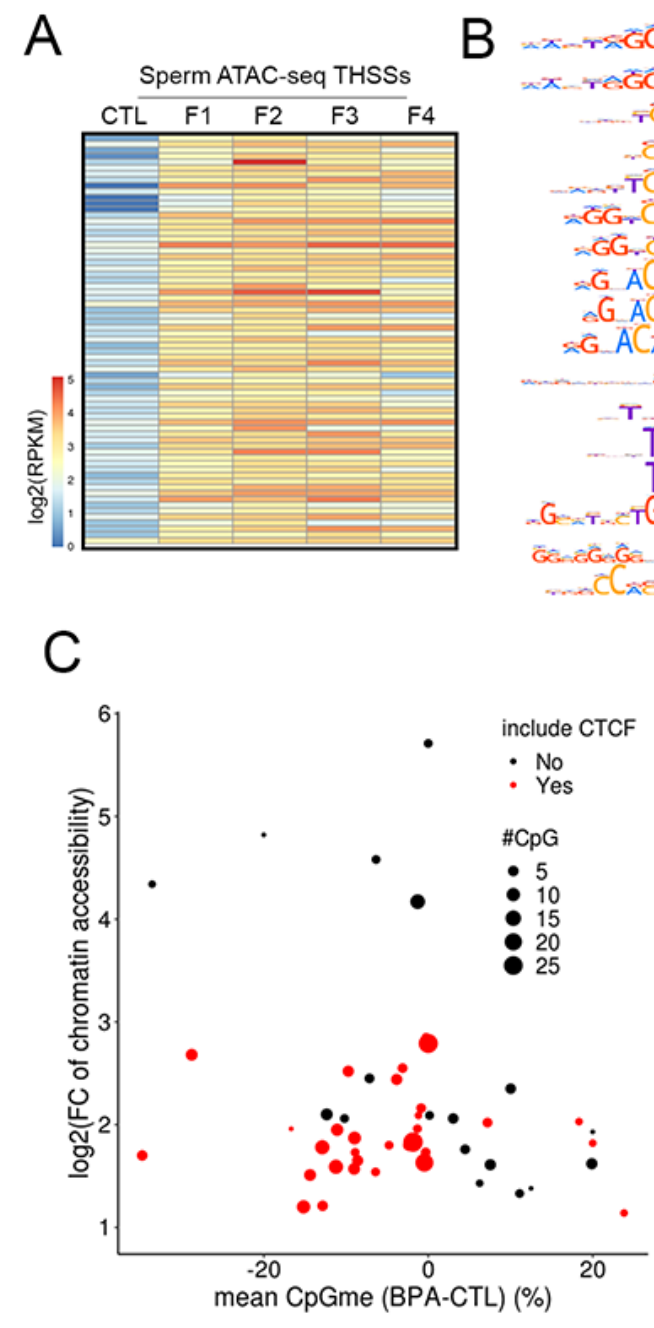

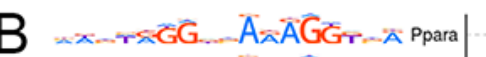
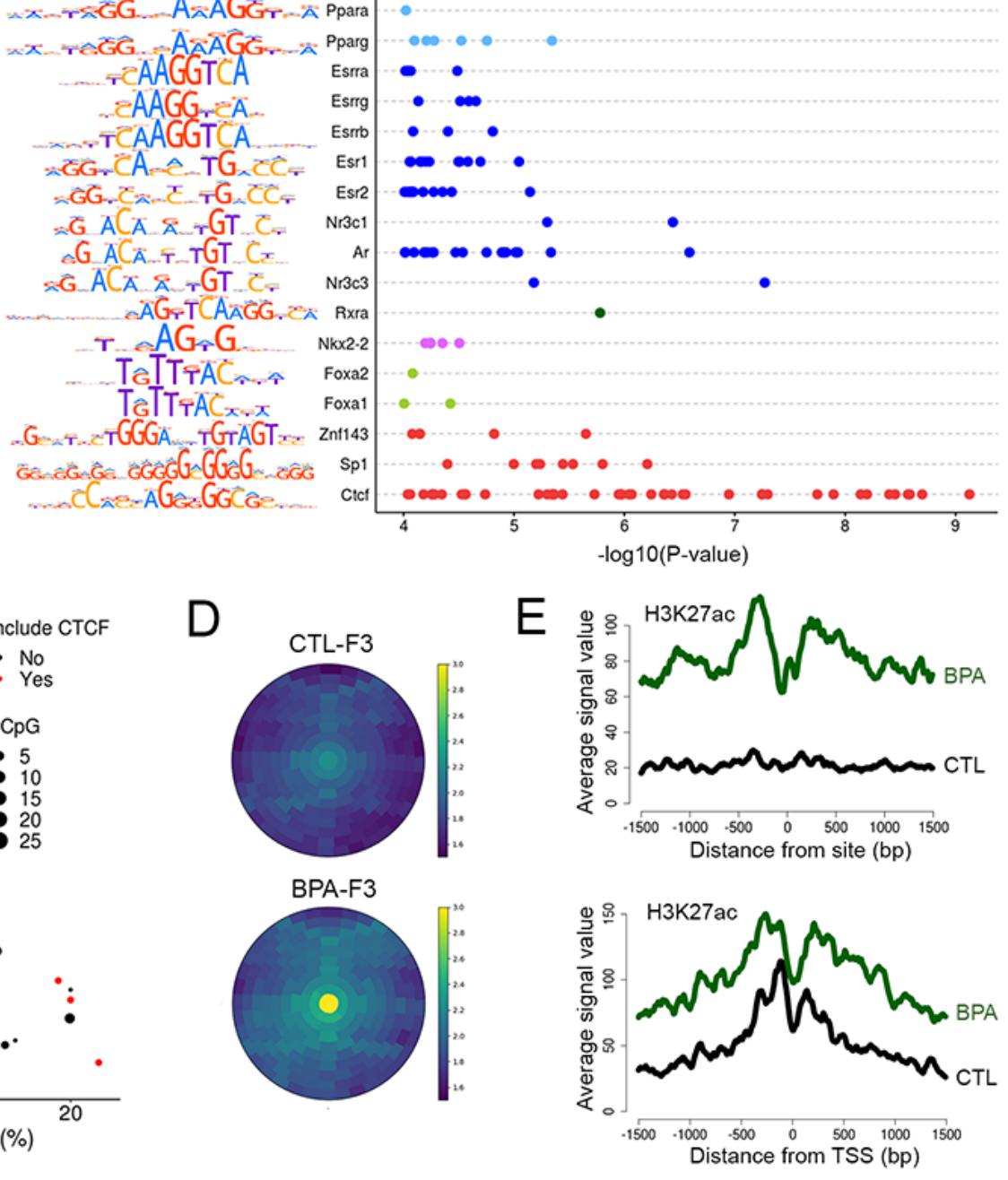

Fig. 3. ATAC-seq accessible sites maintained from the BPA-F1 through the BPA-F4 generations are enriched in CTCF sites.

(A) Heatmap showing ATAC-seq signal at sites present in sperm of BPA-F1-F4 but not controls. (B) TFs whose binding motifs are enriched at ATAC-seq accessible sites present in sperm of BPA-F1 through BPA-F4. (C) Differences in DNA methylation in sperm between BPA-F3 and CTL-F3 at ATAC-seq sites conserved in BPA-F1 through BPA-F4. (D) Interaction frequencies between ATAC-seq sites conserved in sperm of BPA-F1 through BPA-F4 and other sites in the genome calculated using significant interactions from $\mathrm{Hi}-\mathrm{C}$ data obtained in sperm of BPA-F3 and CTL-F3. (E) H3K27ac ChIP-seq signal at differential ATAC-seq sites maintained from BPA-F1 through BPA-F4 (top) and the promoters of the genes they contact (bottom).

frequencies show an increase in contacts between the putative enhancers and the promoters of target genes in BPA-F3 versus control sperm (Fig. 3D). Furthermore, results from ChIP-seq experiments for H3K27ac indicate an increase in the levels of this modification at nucleosomes flanking the 69 enriched sites in BPA-F3 compared to control (Fig. 3E, top) and at the TSSs of upregulated genes contacted by these sites (Fig. 3E, bottom). Of the 610 genes contacted by BPA-induced differential sites, 30 are involved in obesity based on GWAS studies performed in humans (Fig. S4G). Furthermore, mouse carrying mutations in upregulated genes show defects in beta cell physiology, hepatic and adipose tissue morphology, and hormone secretion (Fig. 
$\mathrm{S} 4 \mathrm{H})$. Together, these results suggest that BPA, directly or indirectly, induces the binding of CTCF and various transcription factors at genomic sites that are normally methylated. Many of these sites are hypomethylated with respect to control in the sperm of BPA animals and contain higher levels of H3K27ac. It is possible that these sites correspond to enhancers that become activated in the BPA male germline, as suggested by the presence of H3K27ac. These activated enhancers contact gene promoters at a higher frequency in BPA sperm. As we have previously shown, this information may be transmitted to the embryo after fertilization (11), resulting in alterations in transcription during early embryogenesis.

\section{Activation of an enhancer in the Fto gene in the male and female gametes correlates with transmission of obesity}

Since the obesity epiphenotype starts decreasing in F5, is limited to approximately $60 \%$ of the progeny in F6, and it completely disappears in F7, we examined the correlation between the transmission of the phenotype and the presence of the 69 altered TF sites in sperm from these later generations. Only 12 out of the 69 sites are absent in sperm of F6 compared to F4, suggesting that all or some of these 12 sites, but probably not the rest of the 69 , are responsible for the transgenerational transmission of obesity. We then used significant interactions identified by Fit-Hi-C to ascertain all the genes that interact with these putative enhancers (Table S2) and identified those with the most significant interactions in $\mathrm{Hi}-\mathrm{C}$ data from BPA-F3 with respect to control animals (Fig. S5A). Some of these 12 putative enhancers are located in intergenic regions (Fig. S5B) but others are located in gene introns (Fig. S5C-S5D), and often these enhancers do not interact with the promoter of the gene in which they reside. Rather, they interact with nearby genes, many of which have been shown to be involved in obesity (Table S2). One of these 12 sites is present in intron 8 of the Fto gene (Site 1, Fig. 4A), which encodes a demethylase of N6methyladenosine $\left(\mathrm{m}^{6} \mathrm{~A}\right)$ in RNA. SNPs in the first two introns of this gene in humans have been implicated in obesity based on results from GWAS studies (29). A second site located in an intergenic region interacts strongly with the promoter of the Ythdf3 gene, which encodes an $\mathrm{m}^{6} \mathrm{~A}$ RNA binding protein (Fig. S5A-S5B). Site 1 in intron 8 of Fto contains motifs for the CTCF and Foxa1 proteins. Adjacent to this site, several additional ATAC-seq peaks are either only present in sperm of BPAF1-F5 or their intensity increases in sperm from these animals with respect to BPA-F6 and controls (Sites 2-6, Fig. 4A). These sites were not identified in our original analysis for differential ATAC-seq peaks between BPA-Fi and control because of the stringent statistical conditions imposed in the analysis; however, visual inspection clearly indicates the existence of differential accessibility in a large region of the Fto intron containing Sites 1-6 (Fig. 4A). Interestingly, and in support of a role for Fto in the transgenerational transmission of obesity, these same sites are also accessible in the sperm of male progeny obtained from the embryo transfer experiments (Fig. 4A). These sites contain motifs for CTCF, Esr1, Ar and Ppar $\gamma$. We will refer to this approximately $4 \mathrm{~kb}$ region in the intron of the Fto gene whose accessibility increases in sperm of the male progeny of BPA-exposed females as the "proximal Fto BPA enhancer".

Since the obesity epiphenotype elicited by BPA exposure can also be transmitted through the female germline, alterations in TF binding responsible for obesity should also be present in oocytes. To explore this possibility, we performed ATAC-seq in oocytes from control and BPA-F2 females. Results show that GV oocytes from BPA-F2 females have 478 accessible sites and lack 53 sites with respect to CTL-F2 oocytes. Surprisingly, out of the 12 sites present in BPA-F1-F5 but lost in BPA-F6 sperm (Fig. S5A), only Site 1 present in intron 8 of the Fto gene in sperm of BPA-Fi is also differentially accessible in oocytes (Fig. 4B), suggesting that Fto may be the primary effector of transgenerational inheritance of obesity whereas other differentially accessible sites in the genome may be secondary consequences of Fto activation or unrelated to the obesity phenotype. Sites 2-6 present in intron 8 of Fto in sperm of BPA-Fi males are not present in oocytes 
bioRxiv preprint doi: https://doi.org/10.1101/2020.11.20.391672; this version posted November 20,2020 . The copyright holder for this preprint (which was not certified by peer review) is the author/funder, who has granted bioRxiv a license to display the preprint in perpetuity. It is made available under aCC-BY-NC-ND 4.0 International license.

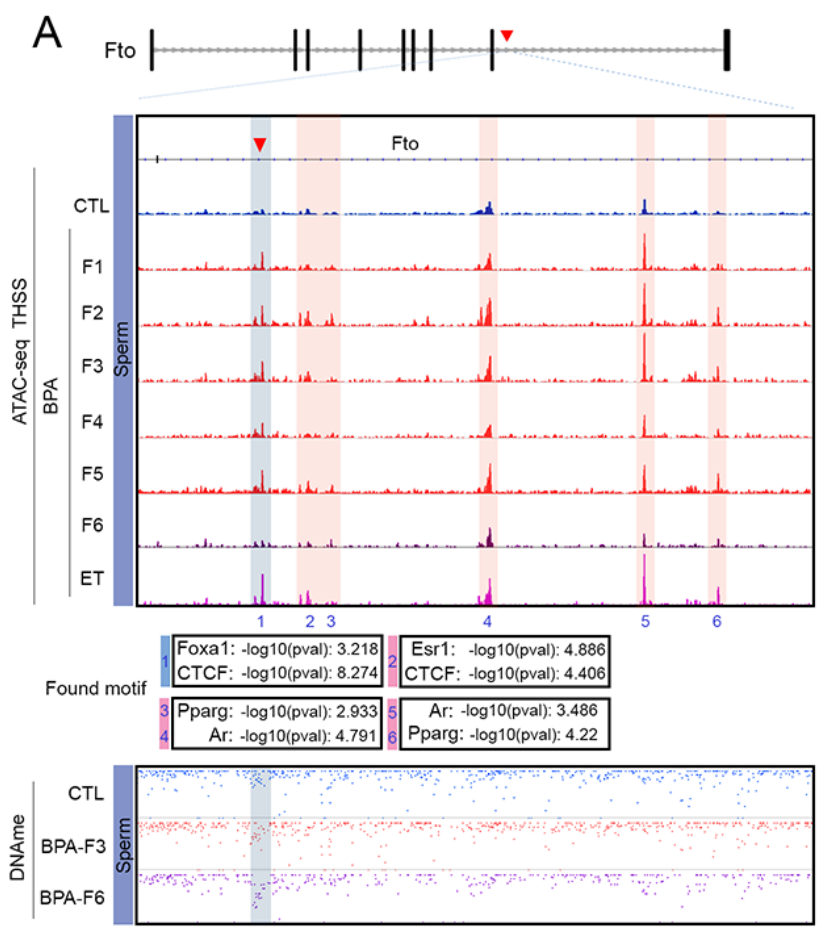

B
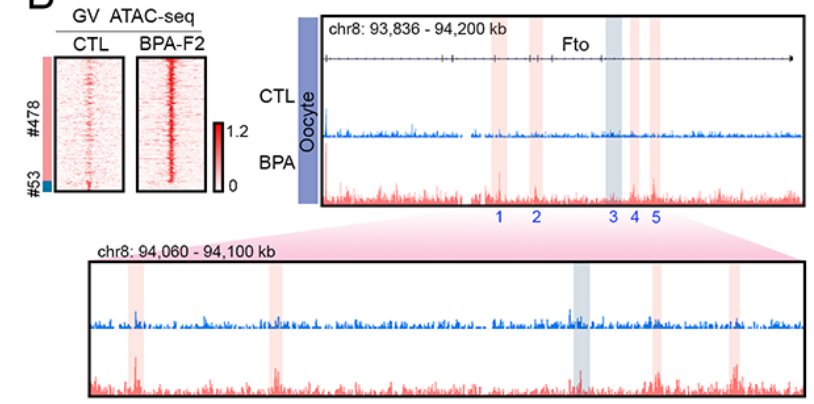

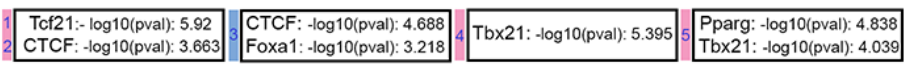

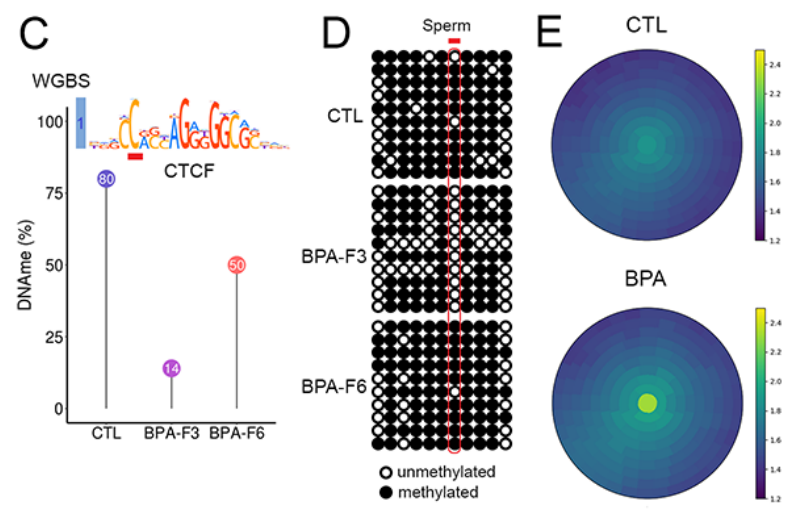

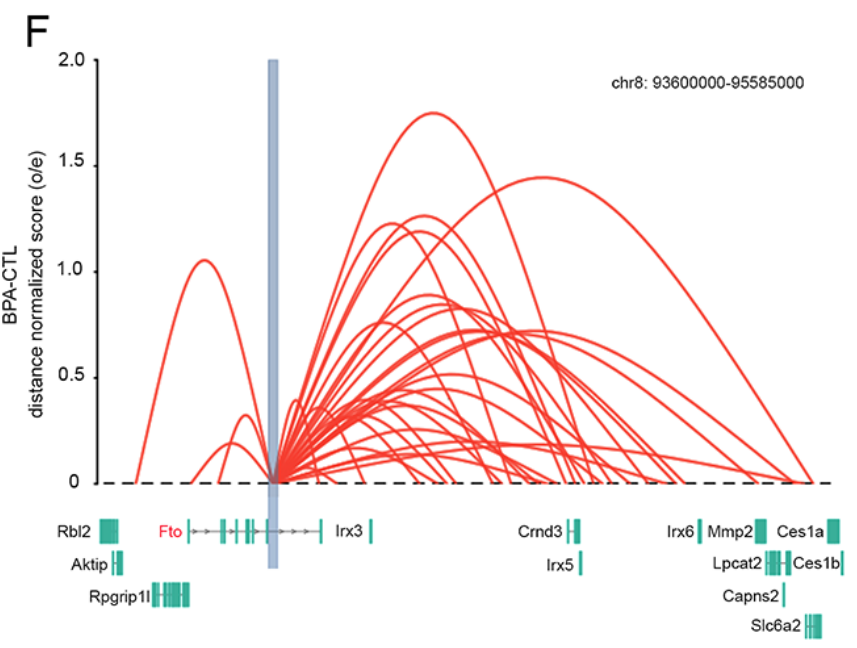
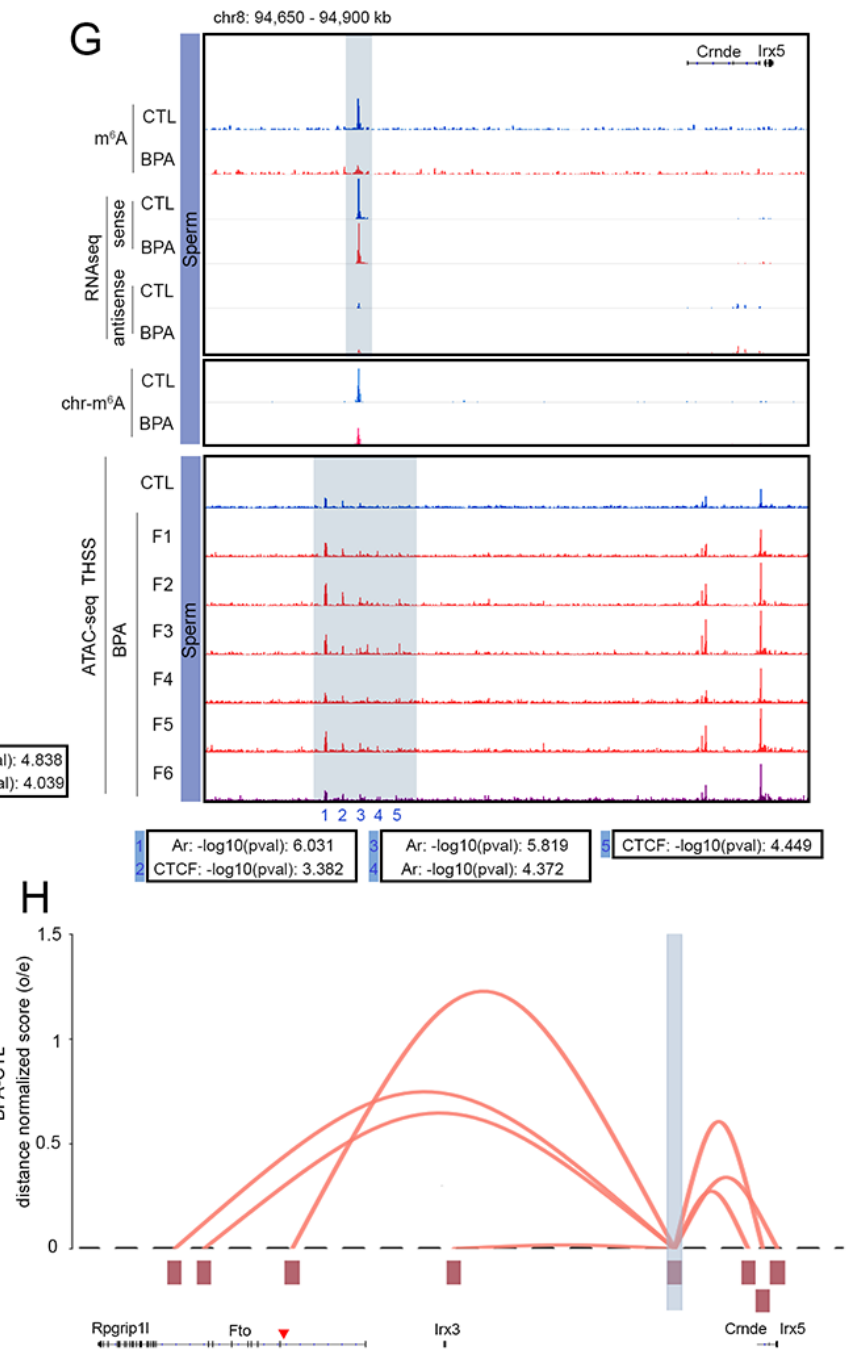
Fig. 4. Changes in accessibility and interactions at a BPA intronic enhancer correlate with transmission of obesity.

(A) Persistence of an ATAC-seq accessible site (Site 1) located in intron 8 of the Fto gene in sperm of BPA-F1 through BPA-F5 males. This site contains motifs for CTCF and Foxa1, and it is also present in the sperm of the male progeny from the embryo transfer (ET) experiment. Other sites in the region are present in CTL and BPA-F6 but they increase in signal in sperm of BPA-F1 through BPA-F5 (top). Site 1 is hypomethylated in BPA-F3 with respect to CTL and BPA-F6 (bottom). (B) ATAC-seq sites in differentially accessible between oocytes of BPA-F2 and CTL-F2. A subset of these sites is located in several introns of Fto and one of them coincides with Site 1 present in intron 8 in sperm. (C) methylation levels at the CpG located in position 2 of the CTCF motif present in Site 1 obtained from WGBS-seq in sperm of CTL, BPA-F3 and BPA-F6. (D) methylation levels at the same CpG but obtained from bisulfite sequencing. (E) differences in interaction frequency between CTL-F3 and BPA-F3 of Site 1 with other sites in the genome obtained from $\mathrm{Hi}-\mathrm{C}$ data using significant interactions. $(\mathbf{F})$ Differential Hi-C interactions between BPA-F3 and CTL-F3 of Site 1 with adjacent genomic sites. (G) RNA-seq and m6A levels in an enhancer RNA present distally from Fto in a region close to the Crnde and Irx5 genes. This putative enhancer contains binding sites for several TFs whose accessibility increases in BPA-Fi with respect to CTL and BPA-F6. (H) The distal enhancer preferentially interacts in sperm of BPA-F3 versus CTL-F3 with the Fto and Irx5 genes.

of BPA-F2 females. Instead, different sites in introns 4, 5, and 8 are accessible in oocytes. These sites contain binding motifs for CTCF, Foxa1, Tcf21, Tbx21 and Ppar $\gamma$. One interesting consequence of results obtained in oocyte of BPA-F2 and those obtained after the embryo transfer experiments using the same BPA-F2 oocytes is that the transcription factor sites present in the male gametes of the embryo transfer progeny have switched from the oocyte to the sperm distribution patterns (Fig. 4A-B).

Site 1 in Fto contains motifs for CTCF and Foxa1, and the disappearance of ATAC-seq signal in BPA-F6 coincides with re-methylation of a differentially methylated region at the site (Fig. $4 A$, lower panel). Since binding of CTCF to DNA is known to be sensitive to the methylation status of a specific C located at position 2 in the core motif (30), we examined the GWBS data for changes in DNA methylation at the CpG located in this position. Results show that the level of methylation in this $\mathrm{CpG}$ is around $80 \%$ in sperm from control males, it decreases to $14 \%$ in sperm from BPA-F3 and increases to $50 \%$ in BPA-F6 sperm (Fig. 4C). To determine the methylation levels of the $\mathrm{CpG}$ present at position 2 in the CTCF motif in Site 1 more quantitatively, we performed bisulfite conversion followed by Sanger sequencing. We find that this $C$ is highly methylated in sperm from control males, is completely demethylated in BPA-F3 sperm and becomes almost completely remethylated at levels similar to control in BPA-F6 sperm (Fig. 4D). Analysis of $\mathrm{Hi}-\mathrm{C}$ data from control and BPA sperm using significant interactions identified using Fit-Hi-C shows an increase in interaction frequency between the Fto BPA enhancer and adjacent sites in the genome, including its own promoter as well as neighboring genes (Fig. 4F). Several of these genes, including Rpgrip1I, Irx3, Irx5, Slc6a2, and Mmp2, have been shown to be involved in the regulation of body size and obesity by affecting appetite and food consumption (31-35), which agrees with observations indicating that BPA-F4 mice have increased food consumption (Fig. 1F). However, the transcript levels for these genes, except for Mmp2, are not statistically significantly different between control and BPA sperm (Fig. S6A). Since sperm are transcriptionally inactive, it is possible that the increased interactions between the Fto BPA enhancer and target promoters are a vestige of previous transcription during germline development and may have a functional effect after fertilization during pre-implantation development (11). 


\section{$\mathrm{m}^{6} \mathrm{~A}$ demethylation of a distal enhancer RNA may also affect Fto expression}

Since Fto encodes an $\mathrm{m}^{6} \mathrm{~A}$ demethylase, we tested whether altered expression of this gene during spermatogenesis affects RNA methylation by measuring $m^{6}$ A levels in sperm RNAs of BPA-F3 and controls. We find a large global decrease in this modification in BPA sperm with respect to control (Fig. S6B). The absence of $\mathrm{m}^{6} \mathrm{~A}$ does not appear to affect stability of sperm RNAs, since levels of both sense and antisense RNAs are similar in BPA-F3 and control (Fig. S6C). Although some of the $\mathrm{m}^{6} \mathrm{~A}$ hypomethylated sites map to exons of mRNAs (Fig. S6D), most are located in introns and intergenic regions, suggesting that they may be present in enhancer RNAs (Fig. S6E) It is possible that the low levels of $\mathrm{m}^{6} \mathrm{~A}$ in sperm RNAs will alter their function during early embryonic development after being transferred to the zygote.

It has been reported recently that $\mathrm{m}^{6} \mathrm{~A}$ hypomethylated eRNAs preferentially localize to chromatin where they increase accessibility (36). Analysis of $\mathrm{Hi}-\mathrm{C}$ data using Fit-Hi-C indicates that putative enhancers in which $\mathrm{m}^{6} \mathrm{~A}$ levels decrease significantly in BPA-F3 with respect to controls interact with promoters of genes involved in various processes including development and response to stimuli (Fig. S6F). We thus examined the region surrounding the Fto locus for putative eRNAs. We noticed the expression of a ncRNA in the region between the Irx3 and Crnde genes (Fig. 4G, top panel; see Fig. 4F for the relative location of these two genes with respect to Fto). This RNA is $\mathrm{m}^{6} \mathrm{~A}$ demethylated in BPA-F3 sperm with respect to control (Fig. 4G, top panel). To further explore the significance of this putative eRNA, we isolated chromosome-associated RNAs from BPA-F3 and control sperm. We then performed RNA-seq and determined $\mathrm{m}^{6} \mathrm{~A}$ levels in chromatin-associated RNAs. Results indicate that the putative eRNA is tightly associated with chromatin and it is hypomethylated in BPA-F3 sperm (Fig. 4G). Interestingly, this RNA is transcribed from a region that is not accessible in control mice, but it becomes accessible in BPAF1-F5 sperm (Fig. 4G, lower panel) and loses accessibility in sperm from the F6 generation. This region was not identified in our previous analyses because the low ATAC-seq signal in the sperm of the F4 generation did not meet the established thresholds (Fig. 4G). The main ATAC-seq peak in the region contains motifs for CTCF and Ar. Other adjacent sites also contain motifs for these two proteins and thus this region could represent an enhancer. Analysis of $\mathrm{Hi}-\mathrm{C}$ data from BPAF3 and control sperm using Fit-Hi-C indicates that this putative enhancer preferentially interacts with the promoter of the Fto, Irx3, and Irx5 genes, as well as the proximal Fto BPA enhancer identified above (Fig. $4 \mathrm{H})$, suggesting that it may regulate the expression of these genes.

\section{Changes in gene expression in visceral fat tissue of BPA-F3 mice}

Since epigenetic alterations induced by BPA are consistently observed in the gametes during generations $\mathrm{F} 1$ through $\mathrm{F} 5$, it is possible that these alterations are present transiently in the early embryo after fertilization, are erased, and then re-established in the germline after gastrulation. However, a more plausible scenario is that these alterations persist at least until the epiblast stage when the germline of the embryo is established. These epigenetic changes may then cause alterations of gene expression in the somatic cells of the embryo that in turn affect the function of adult tissues or may be maintained during development and be present in the tissues of the adult where they exert their effects on gene expression that lead to epiphenotypes. To distinguish between these possibilities, we performed ATAC-seq in several adult tissues, including visceral adipose tissue, adipocyte progenitor cells, hypothalamus, and peripheral blood mononuclear cells (PBMCs). The results show that the Fto proximal BPA enhancer located in intron 8 is activated in these tissues from both control and BPA-F4 animals (Fig. S7A). Interestingly, although the CTCF site found in sperm is also present in all the adult tissues, the pattern of accessible sites indicative of the binding of other TFs is tissue-specific. In agreement with these observations, the expression of Fto and adjacent genes is the same in fat tissue, adipocyte precursors, and hypothalamus from control and BPA-F4 animals (Fig. S7B). These results suggest that the obesity observed in BPA- 

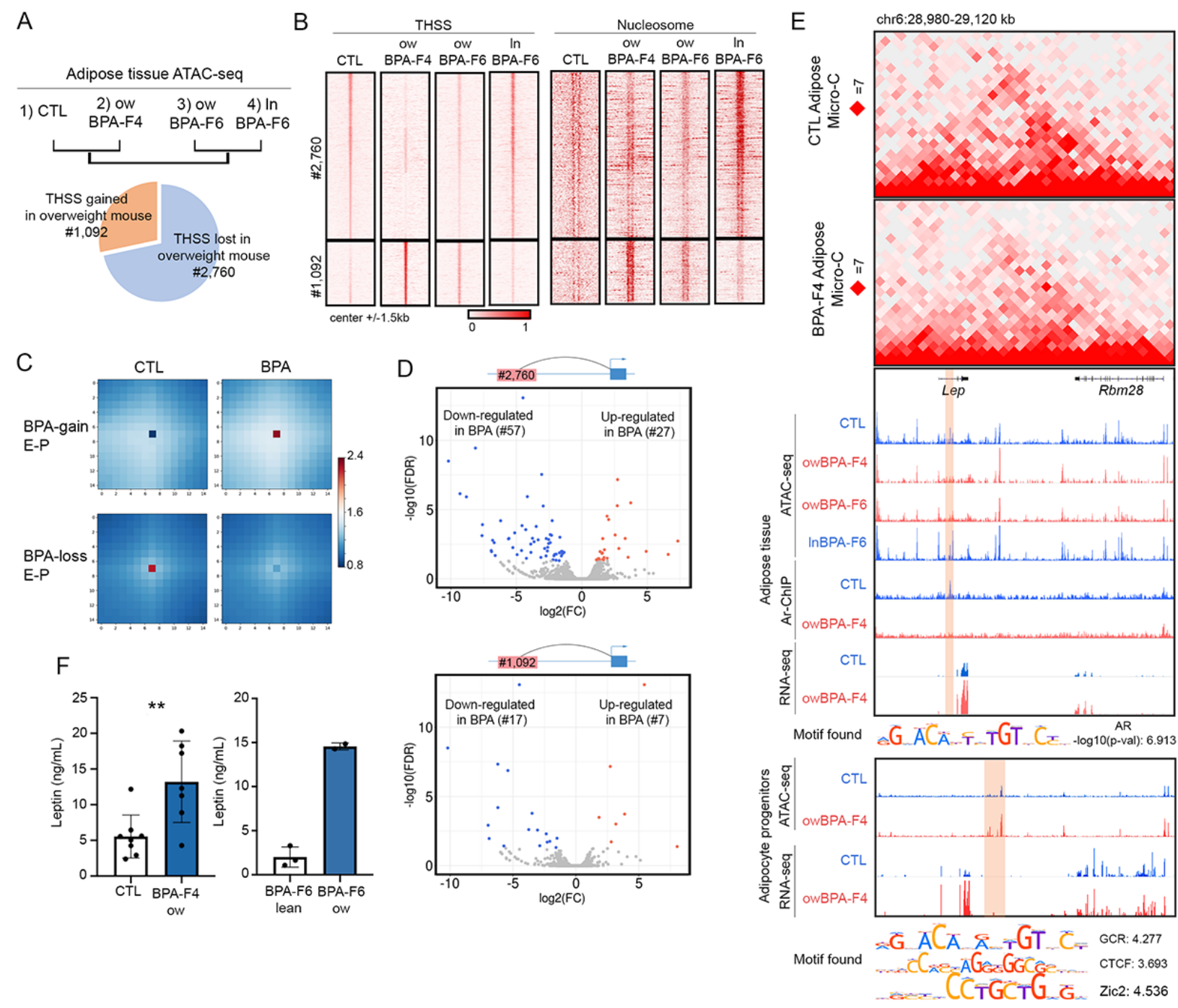

Fig. 5. Changes in chromatin occupancy and 3D interactions in visceral fat tissue.

(A) Summary of differential chromatin accessibility (transposase hypersensitive sites, THSSs) results in visceral adipose tissue from control, BPA-F4, overweight BPA-F6, and lean BPA-F6.

(B) THSSs and flanking nucleosomes with increased or decreased signal in control versus BPA-F4 and BPA-F6 visceral adipose tissue. (C) Increased or decreased interaction frequency between enhancers and promoters located at differential THSSs based on Micro-C data from control and BPA-F4 visceral adipose tissue. (D) Up- and down-regulated interactions between differential THSSs and their target promoters based on Micro-C data from control and BPA-F4 visceral adipose tissue. (E) Micro-C heatmaps, ATAC-seq, RNA-seq, and Ar ChIP-seq data in visceral adipose tissue of control, BPA-F4, and BPA-F6 mice in the region surrounding the leptin gene. The lower panels show ATAC-seq and RNA-seq in the same region from adipocyte precursor cells. (F) Levels of leptin in serum from control, BPA-F4, and lean or overweight BPA-F6 mice.

$\mathrm{Fi}$ animals is not due to miss-expression of Fto in fat tissue. Rather, miss-expression of Fto during embryonic development of BPA-Fi mice must have altered expression of other genes that in turn are responsible for the obesity phenotype in the adult. 
To gain insights into the molecular basis for the obese phenotype, we compared ATAC-seq data obtained in visceral adipose tissue from control, BPA-F4, and lean and over-weight BPA-F6 male mice. We find dramatic changes in the distribution of accessible chromatin sites, with 1,092 sites increased or gained and 2,760 decreased or lost (2-fold change, $p$ value $\leq 0.05$ ) in the adipose tissue of BPA-F4 and overweight BPA-F6 with respect to control and lean BPA-F6 (Fig. 5A and $5 B$ ). These TF sites are flanked by well-positioned nucleosomes, and the changes in TF binding correlate with the intensity of nucleosomal signal (Fig. 5B). TF sites whose binding is altered in adipose tissue are located almost exclusively in intergenic and intronic regions of the genome, suggesting that they may correspond to enhancers (Fig. S7C), and contain motifs for a variety of TFs, including several nuclear hormone receptors (Fig. S7D). None of the altered TF binding sites found in adipose tissue correspond to altered TF sites observed in sperm. To examine whether the altered pattern of TF sites in fat tissue correlate with changes in DNA methylation, we performed GWBS in adipose tissue of control and BPA-F4 animals. Although there is a large number of hypo- and hyper-methylated $\mathrm{CpGs}$ in the genome of BPA-F4 fat tissue with respect to control (Fig. S7E), only 493 hyper-DMRs and 171 hypo-DMRs overlap with sites of altered TF occupancy (Fig. 7SF; see affected TFs in Fig. 7SD). These results suggest that most of the altered TF sites in adipose tissue of BPA-F4 males are unrelated to changes in DNA methylation and vice versa.

To further examine the bases for the obese phenotype, we performed Micro- $C$ using adipose tissue of control and BPA-F4 males (see Table S1 for quality control of Micro-C reads). We then identified significant interactions using Fit-Hi-C and determined those involving TF sites altered in BPA versus control. TF sites gained in BPA-F4 interact with increased frequency with target genes whereas those lost or decreased interact with lower frequency (Fig. 5C). We then performed RNA-seq in adipose tissue of BPA-F4 and control males and identified target genes of altered TF sites that are up- or down-regulated in BPA-F4 adipose tissue with respect to control (Fig. 5D). We also used RNA-seq to identify genes whose expression is altered in adipocyte progenitors obtained from fat tissue of BPA-F4 versus control (Fig. S8A). Results from these experiments indicate that genes up-regulated in adipocyte progenitors of BPA-F4 animals are involved in cell proliferation and various metabolic process (Fig. S8A and S8B), whereas those upregulated in visceral fat tissue are involved in the regulation of cell morphogenesis and cellular protein metabolic process (Fig. S8C and S8D). One of the genes up-regulated in both adipocyte precursors and visceral fat tissue is Lep, which encodes the leptin hormone involved in the regulation of body weight. Leptin is present at higher than normal levels in animals with leptin resistance, leading to increased food consumption (37). Upregulation of Lep in BPA-F4 animals correlates with the disappearance of an accessible region in the intron of the Lep gene. This site, which contains the motif for Ar, is present in fat tissue of control and lean BPA-F6 males but absent in BPA-F4 and overweight BPA-F6 animals (Figure 5E). ChIP-seq with antibodies to Ar confirms the disappearance of Ar in adipose tissue of obese males, and this correlates with a decrease of interactions between Lep and the adjacent Rbm28 gene (Fig. 5E). Interestingly, Lep is also up-regulated in adipocyte progenitors of BPA-F4 where overexpression of this gene correlates with the presence of new binding sites for CTCF, GRC, and Zic2 instead of Ar (Fig. $5 \mathrm{E})$. Increased expression of Lep in adipose tissue correlates with higher levels of the leptin hormone in BPA-F4 and overweight BPA-F6 with respect to control and lean BPA-F6 (Fig. 5F).

\section{Conclusions}

Observations reported here suggest that exposure to BPA results in dramatic alterations of the male and female germline epigenomes, with hundreds of new binding sites for a variety of transcription factors. Although here we have specifically followed those sites that correlate with the transmission of obesity, it is likely that other sites are responsible for the various phenotypes that have been attributed to BPA exposure, including breast and testicular cancers, and autistic- 
like behaviors (38, 39). Many of these sites correspond to nuclear hormone receptors and this may be due to the ability of BPA to bind to these proteins. Thus, it is likely that exposure to different types of environmental perturbations will elicit changes in other classes of transcription factors, resulting in the misregulation of distinct groups of genes and giving rise to different phenotypes. Although at this time we lack direct evidence supporting a direct role for Fto in the transmission and generation of obesity, the strong correlation between the presence of active enhancers interacting with this gene in sperm and oocytes and the inheritance of obesity clearly favors this possibility. The activated Fto enhancers contain binding sites for various nuclear hormone receptors, and it is possible that BPA binds to these receptors in primordial germ cells resulting in their binding to chromatin at the time of the exposure when DNA is normally demethylated. The presence of these factors bound to chromatin may then interfere with DNA remethylation during germline development after E13.5. We have previously shown that a subset of TFs bound to the sperm genome are also found on chromatin during preimplantation development (11). Once the DNA of the embryo, which is demethylated after fertilization, becomes methylated again in the epiblast, sites protected from methylation, where TFs can bind again in differentiated tissues, may not need the continuous presence of bound TFs in order to maintain a memory state of accessible chromatin. Although such a mechanism may explain transgenerational inheritance in general, the involvement of Fto and its role in $\mathrm{m}^{6} \mathrm{~A}$ methylation offers an additional or alternative explanation for the transgenerational transmission of obesity. It is possible that activation of the Fto intronic enhancer by BPA results in lower levels of $\mathrm{m}^{6} \mathrm{~A}$ and preferential persistence of the chromatinassociated eRNA from the intergenic enhancer after fertilization. Since this distal intergenic enhancer interacts with the Fto promoter and its intronic enhancer, this could lead to activation of Fto during preimplantation development. The positive feedback loop between the two enhancers could contribute to the continued expression of the gene and the transmission of this active state between generations. The finding that both genetic mutations and environmentally induced epigenetic alterations in the Fto gene lead to similar phenotypic effects suggest that much of the missing genetic variation required to explain many human diseases may be epigenetic in origin.

\section{References and Notes}

1. J. P. Lim, A. Brunet, Bridging the transgenerational gap with epigenetic memory. Trends Genet 29, 176-186 (2013).

2. L. Daxinger, E. Whitelaw, Understanding transgenerational epigenetic inheritance via the gametes in mammals. Nat Rev Genet 13, 153-162 (2012).

3. O. J. Rando, Intergenerational Transfer of Epigenetic Information in Sperm. Cold Spring Harb Perspect Med 6, (2016).

4. A. Bergman, J. J. Heindel, S. Jobling, K. A. Kidd, R. T. Zoeller. (2015).

5. J. A. Hackett, M. A. Surani, Beyond DNA: programming and inheritance of parental methylomes. Cell 153, 737-739 (2013).

6. U. Brykczynska et al., Repressive and active histone methylation mark distinct promoters in human and mouse spermatozoa. Nature structural \& molecular biology 17, 679-687 (2010).

7. B. R. Carone et al., High-resolution mapping of chromatin packaging in mouse embryonic stem cells and sperm. Developmental cell 30, 11-22 (2014).

8. S. Erkek et al., Molecular determinants of nucleosome retention at CpG-rich sequences in mouse spermatozoa. Nature structural \& molecular biology 20, 868-875 (2013).

9. S. S. Hammoud et al., Distinctive chromatin in human sperm packages genes for embryo development. Nature 460, 473-478 (2009).

10. B. Samans et al., Uniformity of nucleosome preservation pattern in Mammalian sperm and its connection to repetitive DNA elements. Developmental cell 30, 23-35 (2014).

11. Y. H. Jung et al., Maintenance of CTCF- and Transcription Factor-Mediated Interactions from the Gametes to the Early Mouse Embryo. Mol Cell 75, 154-171 e155 (2019). 
12. K. Gapp et al., Implication of sperm RNAs in transgenerational inheritance of the effects of early trauma in mice. Nat Neurosci 17, 667-669 (2014).

13. U. Sharma et al., Biogenesis and function of tRNA fragments during sperm maturation and fertilization in mammals. Science 351, 391-396 (2016).

14. Q. Chen et al., Sperm tsRNAs contribute to intergenerational inheritance of an acquired metabolic disorder. Science 351, 397-400 (2016).

15. A. B. Rodgers, C. P. Morgan, N. A. Leu, T. L. Bale, Transgenerational epigenetic programming via sperm microRNA recapitulates effects of paternal stress. Proc Natl Acad Sci U S A 112, 13699-13704 (2015).

16. P. J. Murphy, S. F. Wu, C. R. James, C. L. Wike, B. R. Cairns, Placeholder Nucleosomes Underlie Germline-to-Embryo DNA Methylation Reprogramming. Cell 172, 993-1006 e1013 (2018).

17. I. Kremsky, V. G. Corces, Transcription factors protect from DNA re-methylation during reprograming of primordial germ cells and pre-implantation embryos. bioRxiv, 850362 (2019).

18. R. Klukovich et al., Environmental Toxicant Induced Epigenetic Transgenerational Inheritance of Prostate Pathology and Stromal-Epithelial Cell Epigenome and Transcriptome Alterations: Ancestral Origins of Prostate Disease. Sci Rep 9, 2209 (2019).

19. I. Sadler-Riggleman et al., Epigenetic transgenerational inheritance of testis pathology and Sertoli cell epimutations: generational origins of male infertility. Environ Epigenet 5, dvz013 (2019).

20. M. Manikkam, C. Guerrero-Bosagna, R. Tracey, M. M. Haque, M. K. Skinner, Transgenerational actions of environmental compounds on reproductive disease and identification of epigenetic biomarkers of ancestral exposures. PLoS One 7, e31901 (2012).

21. W. Dekant, W. Volkel, Human exposure to bisphenol A by biomonitoring: methods, results and assessment of environmental exposures. Toxicol Appl Pharmacol 228, 114134 (2008).

22. L. N. Vandenberg et al., Urinary, circulating, and tissue biomonitoring studies indicate widespread exposure to bisphenol A. Environ Health Perspect 118, 1055-1070 (2010).

23. D. C. Simonson, R. A. DeFronzo, Indirect calorimetry: methodological and interpretative problems. Am J Physiol 258, E399-412 (1990).

24. H. MacKay, A. Abizaid, A plurality of molecular targets: The receptor ecosystem for bisphenol-A (BPA). Horm Behav 101, 59-67 (2018).

25. M. R. Corces et al., An improved ATAC-seq protocol reduces background and enables interrogation of frozen tissues. Nat Methods 14, 959-962 (2017).

26. J. D. Buenrostro, P. G. Giresi, L. C. Zaba, H. Y. Chang, W. J. Greenleaf, Transposition of native chromatin for fast and sensitive epigenomic profiling of open chromatin, DNAbinding proteins and nucleosome position. Nat Methods 10, 1213-1218 (2013).

27. M. J. Rowley et al., Analysis of Hi-C data using SIP effectively identifies loops in organisms from C. elegans to mammals. Genome Res, (2020).

28. H. M. Xu et al., Expression and clinical significance of obesity-associated gene STEAP4 in obese children. Genet Mol Res 15, (2016).

29. R. J. Loos, G. S. Yeo, The bigger picture of FTO: the first GWAS-identified obesity gene. Nat Rev Endocrinol 10, 51-61 (2014).

30. H. Hashimoto et al., Structural Basis for the Versatile and Methylation-Dependent Binding of CTCF to DNA. Mol Cell 66, 711-720 e713 (2017).

31. J. F. M. Carli, C. A. LeDuc, Y. Zhang, G. Stratigopoulos, R. L. Leibel, The role of Rpgrip1I, a component of the primary cilium, in adipocyte development and function. FASEB J 32, 3946-3956 (2018). 
32. J. I. Bjune et al., The homeobox factor Irx3 maintains adipogenic identity. Metabolism 103, 154014 (2020).

33. J. I. Bjune et al., IRX5 regulates adipocyte amyloid precursor protein and mitochondrial respiration in obesity. Int J Obes (Lond) 43, 2151-2162 (2019).

34. R. Mazor et al., Cleavage of the leptin receptor by matrix metalloproteinase-2 promotes leptin resistance and obesity in mice. Sci Trans/ Med 10, (2018).

35. R. M. Pirzgalska et al., Sympathetic neuron-associated macrophages contribute to obesity by importing and metabolizing norepinephrine. Nat Med 23, 1309-1318 (2017).

36. J. Liu et al., $\mathrm{N}(6)$-methyladenosine of chromosome-associated regulatory RNA regulates chromatin state and transcription. Science 367, 580-586 (2020).

37. N. Sainz, J. Barrenetxe, M. J. Moreno-Aliaga, J. A. Martinez, Leptin resistance and dietinduced obesity: central and peripheral actions of leptin. Metabolism 64, 35-46 (2015).

38. J. T. Wolstenholme et al., Transgenerational Bisphenol A Causes Deficits in Social Recognition and Alters Postsynaptic Density Genes in Mice. Endocrinology 160, 18541867 (2019).

39. M. Manikkam, R. Tracey, C. Guerrero-Bosagna, M. K. Skinner, Plastics derived endocrine disruptors (BPA, DEHP and DBP) induce epigenetic transgenerational inheritance of obesity, reproductive disease and sperm epimutations. PLoS One 8 , e55387 (2013).

40. M. Hisano et al., Genome-wide chromatin analysis in mature mouse and human spermatozoa. Nature protocols 8, 2449-2470 (2013).

41. N. Venisse et al., Development and validation of an LC-MS/MS method for the simultaneous determination of bisphenol $A$ and its chlorinated derivatives in adipose tissue. Talanta 204, 145-152 (2019).

42. S. S. Rao, J. J. Lannutti, M. S. Viapiano, A. Sarkar, J. O. Winter, Toward 3D biomimetic models to understand the behavior of glioblastoma multiforme cells. Tissue Eng Part $B$ Rev 20, 314-327 (2014).

43. B. Langmead, S. L. Salzberg, Fast gapped-read alignment with Bowtie 2. Nature methods 9, 357-359 (2012).

44. T. Liu, Use model-based Analysis of ChIP-Seq (MACS) to analyze short reads generated by sequencing protein-DNA interactions in embryonic stem cells. Methods Mol Biol 1150, 81-95 (2014).

45. J. Piper et al., Wellington-bootstrap: differential DNase-seq footprinting identifies celltype determining transcription factors. BMC Genomics 16, 1000 (2015).

46. S. S. Rao et al., A 3D map of the human genome at kilobase resolution reveals principles of chromatin looping. Cell 159, 1665-1680 (2014).

47. F. Ay, T. L. Bailey, W. S. Noble, Statistical confidence estimation for Hi-C data reveals regulatory chromatin contacts. Genome Res 24, 999-1011 (2014).

Acknowledgements: We would like to thank Dr. Cynthia Vied at the Translational Science Laboratory of Florida State University for help with Illumina Sequencing; Drs. Karolina PiotrowskaNitsche and Christopher Raymond from the Emory Mouse Transgenic and Gene Targeting Core for their assistance with the embryo transfer; and Dr. Fred Strobel from the Emory Mass Spectrometry Core for his assistance with detection of BPA in mouse embryos. Funding: This work was supported by U.S. Public Health Service Awards R01 ES027859 (V.G.C.) and R35 NS111602, R01 HG008935, and U01 MH116441 (P.J.) from the National Institutes of Health. BJB was supported by NIH T32 GM008490. The content is solely the responsibility of the authors and does not necessarily represent the official views of the National Institutes of Health. Author Contributions: YHJ planned and performed experiments, analyzed data, and contributed to writing the manuscript; $\mathrm{H}$-LW performed experiments; HL performed experiments; BJB performed experiments and analyzed data; AMS performed experiments; J-FX performed experiments; DR 
bioRxiv preprint doi: https://doi.org/10.1101/2020.1120.391672; this version posted November 20,2020 . The copyright holder for this preprint

(which was not certified by peer review) is the author/funder, who has granted bioRxiv a license to display the preprint in perpetuity. It is made available under aCC-BY-NC-ND 4.0 International license.

performed experiments and analyzed data; PJ planned experiments; VGC planned experiments and wrote the manuscript. Competing Interests: The authors declare no competing interests. Data and Materials Availability: ChIP-seq, ATAC-seq, RNA-seq, $\mathrm{m}^{6} \mathrm{~A}$ and Hi-C data are available from NCBl's Gene Expression Omnibus (GEO). The accession number for all the datasets reported in this paper is GSE149309. Reviewers can access these data using token mjwvqswoljmbziz. Custom scripts were used to separate ATAC-seq reads into subnucleosomal and nucleosome-size ranges. These scripts are available without restrictions upon request.

\section{List of Supplemental Materials}

Materials and Methods

Fig. S1-S8

Tables S1-S2 
bioRxiv preprint doi: https://doi.org/10.1101/2020.1120.391672; this version posted November 20,2020 . The copyright holder for this preprint

(which was not certified by peer review) is the author/funder, who has granted bioRxiv a license to display the preprint in perpetuity. It is made available under aCC-BY-NC-ND 4.0 International license.

\section{Supplemental Materials for \\ Transgenerational inheritance of BPA-induced obesity correlates with transmission of new CTCF sites in the Fto gene}

Yoon Hee Jung ${ }^{1}$, Brianna J. Bixler ${ }^{1}$, Daniel Ruiz', Hsiao-Lin V. Wang ${ }^{1}$, Hannah Linsenbaum ${ }^{1}$, Jian-Feng Xiang ${ }^{1}$, Andrew M. Shafik ${ }^{1}$, Peng Jin ${ }^{1}$, and Victor G. Corces ${ }^{1}$

\section{This pdf file includes}

Materials and Methods

Fig. S1-S8

Tables S1-S2 


\section{Materials and Methods}

\section{Experimental Model and Subject Details}

Mice were maintained and handled in accordance with the Institutional Animal Care and Use policies at Emory University. All experiments were conducted according to the animal research guidelines from $\mathrm{NIH}$ and all protocols for animal usage were reviewed and approved by the Institutional Animal Care and Use Committee (IACUC). Mice were housed in standard cages on a 12: $12 \mathrm{~h}$ light:dark cycle and given ad lib access to food and water. Healthy 8-week old CD1 mice (Charles River Labs) not involved in previous procedures were used for all experiments. Gestating females (F0) were administered daily intraperitoneal injections of Bisphenol A (Sigma $239658,50 \mathrm{mg} / \mathrm{kg}$ ) or sesame oil (Sigma S3547) to prevent irritation at the injection site. Injections were performed from embryonic day 7.5 through 13.5. No sibling breeding was used to avoid inbreeding artifacts.

\section{Isolation of mouse sperm and oocytes}

Euthanasia was performed by $\mathrm{CO}_{2}$ asphyxiation and the epididymis was removed. Mature sperm were collected from the dissected cauda epididymis of 8-10-week-old CD1 mice (Charles River Labs). After dissection to eliminate blood vessels and fat, the cauda epididymis was rinsed with PBS, deposited in Donners medium in a cell culture plate, and punctured with a needle. Sperm were then transferred to a tube and allowed to swim up for $1 \mathrm{hr}$ (40). Purity of sperm was determined by examination under a microscope after DAPI staining. After counting $1000 \mathrm{sperm}$, purity was determined to be at least $99.9 \%$ if no contaminating cells were observed. Ovaries were harvested from 16-day-old CD1 female pups for the preparation of GV-stage oocytes. The zona pellucida was removed with hyaluronidase to avoid any residual cumulus cells.

\section{Isolation of adipocyte progenitor cells}

Visceral adipose tissue (1g) was minced manually in PBS (Ca2+ and $\mathrm{Mg} 2+$ free), transferred to $10 \mathrm{ml}$ of DMEM/F12 containing $0.8 \%$ fatty acid-free bovine serum albumin, collagenase $\mathrm{D}(1.5$ units $/ \mathrm{ml}$; Roche), and dispase II (2.4 units $/ \mathrm{ml}$; Roche), and then incubated at $37^{\circ} \mathrm{C}$ with agitation for $45 \mathrm{~min}$. The reaction was quenched with DMEM/F12 containing 10\% FBS and dissociated cells were passed through a $100 \mu \mathrm{m}$ filter. After centrifugation, the pellet was resuspended in red blood lysis buffer and incubated for 5 min at RT. The reaction was quenched with DMEM/F12 containing 10\% FBS and passed through a $40 \mu \mathrm{m}$ filter. After recovering cells in chilled MACS buffer, adipocyte progenitor cells were isolated using the Adipose Tissue Progenitor Isolation Kit, mouse (Miltenyi Biotec, 130-106-639) according to the manufacturer's protocol.

\section{In vitro fertilization (IVF) and embryo transfer}

IVF was performed using BPA-F2 MII oocytes and epididymal sperm from control males. In brief, superovulation was induced in BPA-F2 CD1 females at 6-8 weeks of age using intraperitoneal injection of $7.5 \mathrm{IU}$ of pregnant mare serum gonadotropin (PMSG) followed by $5 \mathrm{IU}$ of human chorionic gonadotropin (hCG) at $48 \mathrm{~h}$ post-PMSG injection. MII oocytes were harvested at 13-14 $\mathrm{h}$ post-hCG injection. Sperm collected from the cauda epididymis were transferred into droplets containing oocytes. After 3-4 h of co-incubation, oocytes were freed from sperm and cumulus cells using a fine glass pipette. For embryo transfer experiments, two-cell embryos produced by IVF were transferred into the oviducts of day 1 pseudopregnant control CD1 females mated with a vasectomized male.

\section{Indirect calorimetry and food intake}

Control- and BPA-F4 CD1 mice ( $\mathrm{n}=7-13$ per group) were singly housed in metabolic cages (Columbus Instruments CLAMS-HC) and maintained under otherwise standard housing conditions (12-hour light-dark cycle; $22.2 \pm 1.1^{\circ} \mathrm{C}$ ) for indirect calorimetry and food intake assessments. After a 2-day acclimation period, $\mathrm{O}_{2}$ consumption, $\mathrm{CO}_{2}$ production, and food intake 
were monitored over 14-minute periods for consecutive light-dark cycles over 3 successive days. The respiratory exchange ratio (RER) was calculated as the ratio of $\mathrm{CO}_{2}$ production over $\mathrm{O}_{2}$ consumption in 14-minute intervals. Mice were provided ad libitum food access, and food intake was averaged for each mouse during each 12-hour cycle (light or dark).

\section{Determination of BPA levels by mass spectrometry}

F0 pregnant females were injected with $50 \mathrm{mg} / \mathrm{kg}$ of BPA or sesame oil vehicle every $24 \mathrm{~h}$ from E7.5 to E13.5 of fetal development. Embryos from pregnant females were collected $10 \mathrm{~min}, 2 \mathrm{~h}$, and $24 \mathrm{~h}$ post injection on E13.5. From each timepoint, $1 \mathrm{~g}$ of embryo was pooled, homogenized in $500 \mu \mathrm{l}$ of water, and BPA was extracted as described (41). Briefly, $0.25 \mathrm{mg}$ of BPA-d16 was spiked in as an internal standard, BPA was extracted with $500 \mu \mathrm{l}$ of acetonitrile, quenched with $100 \mathrm{mg}$ of MIX I and $100 \mathrm{mg}$ of MIX IV (41). The upper organic layer was then collected and evaporated under a stream of nitrogen gas. The precipitate was dissolved in $200 \mu$ of water/methanol (70/30 v/v). Negative ion LTQ-FTMS was run with isocratic $50 \%$ acetonitrile and $50 \% 20 \mathrm{mM}$ ammonium formate/water solution, with a retention time of 3.1 minutes and $5 \mu \mathrm{g} / \mathrm{ml}$ limit of detection.

\section{Lipid droplet fluorescent assay}

Visceral adipose tissue was fixed for $30 \mathrm{~min}$ in $4 \%$ paraformaldehyde, washed with PBS, and stained with HCS LipidTox detection reagent (Invitrogen H34476). Stained tissues were then photographed under a Zeiss Axio Observer Z1 microscope.

\section{Assay for transposase-accessible chromatin using sequencing (ATAC-seq)}

ATAC-seq was carried out using the Omni-ATAC protocol (25). After sperm cells were counted, the nuclei from 100,000 sperm were isolated with Lysis Buffer $(10 \mathrm{mM}$ Tris- $\mathrm{HCl} \mathrm{pH} \mathrm{7.4,10} \mathrm{mM}$ $\mathrm{NaCl}, 3 \mathrm{mM} \mathrm{MgCl} 2$ ) containing $0.1 \%$ NP40, 0.1\% Tween-20, and 0.01\% digitonin. The purified sperm nuclei pellet was then resuspended in the transposase reaction mix containing $0.05 \%$ digitonin and incubated for $30 \mathrm{~min}$ at $37^{\circ} \mathrm{C}$. To perform ATAC-seq using GV oocytes, around 500 oocytes were deposited in a tube with $500 \mu$ of RSB $(10 \mathrm{mM}$ Tris- $\mathrm{HCl}, 10 \mathrm{mM} \mathrm{NaCl}$ and $3 \mathrm{mM}$ $\mathrm{MgCl}_{2}$ ), and spun down at 500 rcf for $5 \mathrm{~min}$. After careful removal of the supernatant, $15 \mu \mathrm{l}$ of $2 \mathrm{x}$ TD buffer, $0.75 \mu$ l of Tn5, $0.3 \%$ of $1 \%$ Digitonin, $0.3 \%$ of $10 \%$ Tween20 and $0.3 \mu \mathrm{l}$ of $10 \%$ NP40 were added and samples were incubated for $30 \mathrm{~min}$ at $37^{\circ} \mathrm{C}$. Following incubation, sperm or oocytes were treated with Proteinase $\mathrm{K}$ at $55^{\circ} \mathrm{C}$ for $2 \mathrm{~h}$, and gDNA was isolated by phenol:chloroform:isoamyl alcohol and $\mathrm{EtOH}$ precipitation. Library amplification was done with $2 \mathrm{x}$ KAPA HiFi mix (Kapa Biosystems) and $1.25 \mu \mathrm{M}$ indexed primers using the following PCR conditions: $72^{\circ} \mathrm{C}$ for $5 \mathrm{~min} ; 98^{\circ} \mathrm{C}$ for $30 \mathrm{~s}$; and $10-11$ cycles at $98^{\circ} \mathrm{C}$ for $10 \mathrm{~s}, 63^{\circ} \mathrm{C}$ for $30 \mathrm{~s}$, and $72^{\circ} \mathrm{C}$ for $1 \mathrm{~min}$.

\section{Bisulfite Sanger sequencing}

DNA was extracted from epididymal sperm using phenol/chloroform/isoamylalcohol (25:24:1). About 2 ug of DNA was bisulfite converted and purified using the Zymo EZ DNA methylation lightning kit (D5030) following the manufacturer's protocol. Bisulfite converted DNA was then used as template for PCR amplification with KAPA HiFi HotStart Uracil kit (Roche, KK2801) using primers surrounding Site 1 in intron 8 of the Fto gene. The resulting 470 bp fragment was purified using the Zymoclean Gel DNA Recovery kit (D4008). The primer sequences used were as follows: forward 5'- TGGAGTAGGYGTTTTGAGGTGAAAGGGTAG-3' and reverse, 5'TCACTACRATTTTTCCTAACATAACAAAC-3'. Amplicons were ligated to T-Vector pMD19 (Takara, No.3271) with T4 ligase (NEB, M0202L) and transformed into DH-5a competent cells. DNA was isolated from monoclonal bacterial colonies and sequenced.

\section{Whole genome bisulfite sequencing}


DNA $(1 \sim 2 \mu \mathrm{g})$ was isolated from cauda epididymis sperm using phenol:chloroform:isoamyl alcohol followed by EtOH precipitation and spiked with $0.5 \%$ unmethylated lambda genomic DNA (Promega) to determine conversion efficiency after bisulfite treatment. The DNA was sheared with a Diagenode Bioruptor to yield DNA fragments ranging from 200 to $500 \mathrm{bp}$. After purification of the fragmented DNA using phenol/chloroform, DNA fragments were end repaired, A-tailed, and ligated to methylated Illumina adaptors. The Zymo EZ DNA methylation lightning kit (D5030) was used for bisulfite conversion and subsequent purification according to the manufacturer's instructions. Two independent biological replicates per sample were then sequenced using an Illumina NovaSeq 6000 instrument.

\section{ChIP-seq with H3K27ac}

Chromatin immunoprecipitation to detect histone modifications was carried out as described (40) using antibodies to H3K27ac (ab4729, Abcam). In brief, $5 \times 10^{6}$ sperm cells were digested with MNase $\left(15\right.$ units $\left./ 2 \times 10^{6}\right)$ at $37^{\circ} \mathrm{C}$ in a thermomixer for $5 \mathrm{~min}$ and the reaction was quenched with $0.5 \mathrm{M}$ EDTA. Chromatin was pre-cleared with 1:1 protein A:protein G Dynabeads (Life Technologies) and immunoprecipitation was carried out with $\mathrm{H} 3 \mathrm{~K} 27 \mathrm{ac}$-bead complexes at $4^{\circ} \mathrm{C}$ overnight. Libraries for Illumina sequencing were constructed using the following standard protocol. Fragment ends were repaired using the NEBNext End Repair Module and adenosine was added at the 3' ends using Klenow fragment (3' to 5' exo minus, New England Biolabs). Precipitated DNA was incubated with adaptors at room temperature for $1 \mathrm{~h}$ with T4 DNA ligase (New England Biolabs) and amplified with Illumina primers.

\section{ChIP-seq with Ar}

5 million sperm cells were crosslinked with $1 \%$ formaldehyde in 1 XPBS for 10 min at RT, and the reaction was quenched with $125 \mathrm{mM}$ glycine for 10 min at RT. After washing with PBS, cells were lysed with $5 \mathrm{mM}$ PIPES, $85 \mathrm{mM} \mathrm{KCl}, 0.5 \%$ NP40 and 1x protease inhibitors (P8107S, NEB) on ice for 15 min. After centrifugation, sperm cells were resuspended in RIPA buffer (1x PBS; $1 \%$ NP40; $0.5 \%$ sodium deoxycholate; $0.1 \%$ SDS; $1 \times$ protease inhibitors) and incubated on ice for 20 min. The purified sperm chromatin was sonicated to 300-500 bp using a Diagenode Bioruptor. After 25 cycles (30 seconds on and 60 seconds off), the supernatant containing sheared chromatin was collected. Immunoprecipitation was performed overnight at $4^{\circ} \mathrm{C}$ with antibody to the androgen receptor (sc-816, Santa Cruz). Libraries for Illumina sequencing were constructed using the following standard protocol. Fragment ends were repaired using the NEBNext End Repair Module and adenosine was added at the 3' ends using Klenow fragment (3' to 5' exo minus, New England Biolabs). Precipitated DNA was incubated with adaptors at room temperature for $1 \mathrm{hr}$ with T4 DNA ligase (New England Biolabs) and amplified with Illumina primers.

\section{RNA-seq}

Total RNA was isolated from epididymal sperm using Trizol reagent (Invitrogen) and ribosomal RNA was removed using the RiboMinus Transcrptome isolation kit (Invitogen, K1550). RNA concentration was measured using the Qubit RNA HS Assay kit (Thermo Fisher) and fragmented randomly by adding fragmentation buffer. cDNA was synthesized using RNA template and random hexamer primers. After terminal repair, A ligation and sequencing adaptor ligation, the double-stranded cDNA library was completed by size selection and PCR enrichment. Illumina libraries were sequenced paired-end 50 bp on a NovaSeq 6000 instrument.

\section{MeRIP-seq}

Total RNA from sperm was isolated as described above. For $m^{6} \mathrm{~A}$ immunoprecipitation, purified RNA was digested using RNA Fragmentation Reagents (Ambion AM8740) by incubation at $70^{\circ} \mathrm{C}$ for $5 \mathrm{~min}$ in fragmentation buffer and precipitated via standard ethanol precipitation. Anti- $\mathrm{m}^{6} \mathrm{~A}$ antibody (Synaptic Systems Cat.no 202 003) was incubated with $30 \mu$ of Dynabeads Protein A 
for $1 \mathrm{~h}$ at $4^{\circ} \mathrm{C}$. Fragmented RNA was incubated with antibody-bead mixture overnight at $4^{\circ} \mathrm{C}$. Library preparation was performed by using the SMARTer Stranded Total RNA-seq kit v2 (Takara) according to the manufacturer's protocol.

\section{Chromosome-associated RNA m $^{6}$ A-seq}

Fractionation of chromosome-associated RNAs and generation of $\mathrm{m}^{6} \mathrm{~A}$-seq libraries were carried out as described (36). Briefly, $10^{8}$ sperm cells were isolated from the cauda epididymis and resuspended in cold lysis buffer $(10 \mathrm{mM}$ Tris- $\mathrm{HCl}, 0.05 \% \mathrm{NP} 40,150 \mathrm{mM} \mathrm{NaCl})$. After incubation on ice for $5 \mathrm{~min}$, cells were resuspended with 2.5 volumes of sucrose solution (24\% in lysis buffer), and then centrifuged at $4^{\circ} \mathrm{C}$ at $14,000 \mathrm{~g}$ for $10 \mathrm{~min}$. The supernatant (cytoplasmic fraction) was removed and the nuclei pellet was resuspended in glycerol buffer $(20 \mathrm{mM}$ Tris- $\mathrm{HCl}, 75 \mathrm{mM} \mathrm{NaCl}$, $0.5 \mathrm{mM}$ EDTA, $0.85 \mathrm{mM}$ DTT, $0.125 \mathrm{mM}$ PMSF, $50 \%$ glycerol) and the same volume of cold nuclei lysis buffer (10 mM HEPES, $10 \mathrm{mM}$ DTT, $7.5 \mathrm{mM} \mathrm{MgCl}$, $0.2 \mathrm{mM}$ EDTA, $0.3 \mathrm{M} \mathrm{NaCl}, 1 \mathrm{M}$ urea, $1 \%$ NP40). After resuspending the pellet by vortexing, the nuclei were incubated on ice for $2 \mathrm{~min}$ and centrifuged at $4^{\circ} \mathrm{C}$ at $14,000 \mathrm{~g}$ for $2 \mathrm{~min}$. The supernatant (soluble nuclear fraction/nucleoplasm) were removed and the chromosome-associated fraction was collected in the pellet. Total RNA from the chromosome-associated fraction was isolated with Trizol. Ribosomal RNA was removed using the RiboMinus transcriptome isolation kit (Invitrogen, K1550), and then RNA was digested using RNA Fragmentation Reagents (Ambion AM8740) by incubation at $70^{\circ} \mathrm{C}$ for $5 \mathrm{~min}$. $\mathrm{m}^{6} \mathrm{~A}$-immunoprecipitaion was performed using the EpiMark N6Methyladenosine Enrichment Kit (NEB E1610S), followed by library preparation using the SMARTer Stranded Total RNA-seq kit v2 (Takara) according to the manufacturer's protocols.

\section{In-situ Hi-C}

in-situ Hi-C libraries were prepared using Dpnll restriction enzyme as previously described (42). Briefly, 10 million sperm were crosslinked with $1 \%$ formaldehyde, quenched with glycine, washed with PBS, and permeabilized to obtain intact nuclei. Nuclear DNA was then digested with DpnII, the 5'-overhangs were filled with biotinylated dCTPs and dA/dT/dGTPs to make blunt-end fragments, which were then ligated, reverse-crosslinked, and purified by standard DNA ethanol precipitation. Purified DNA was sonicated to 200-500 bp small fragments and captured with streptavidin beads. Standard Illumina TruSeq library preparation steps, including end-repairing, A-tailing, and ligation with universal adaptors were performed on beads, washing twice in Tween Washing Buffer (5mM Tris-HCl pH 7.5, 0.5mM EDTA, $1 \mathrm{M} \mathrm{NaCl}, 0.05 \%$ Tween 20) between each step. DNA on the beads was PCR amplified with barcoded primers using KAPA SYBR FAST qPCR Master Mix (Kapa Biosystems) for 5 12 PCR cycles to obtain enough DNA for sequencing. Generated libraries were paired-end sequenced on an Illumina NovaSeq 6000 instrument. Two biological replicates were generated, and replicates were combined for all analyses after ensuring high correlation.

\section{Micro-C}

Micro-C libraries were prepared as described (Hsieh et al., 2016, Nature methods). Briefly, for each replicate library, 2 million cells obtained from visceral adipose tissue were crosslinked with $1 \%$ formaldehyde for $10 \mathrm{~min}$ at room temperature. Formaldehyde crosslinking was quenched with $0.25 \mathrm{M}$ glycine for $5 \mathrm{~min}$ at RT. After washing with 1xPBS, resuspended cells were further crosslinked with $3 \mathrm{mM}$ DSG in PBS for $40 \mathrm{~min}$ at RT. Crosslinking was quenched with $0.4 \mathrm{M}$ glycine for $5 \mathrm{~min}$ at RT. Cells were resuspended in cold 1x PBS and incubated on ice for $20 \mathrm{~min}$. After collecting cells by centrifugation, cells were washed with buffer $\mathrm{MB \# 1}$ (10 mM Tris- $\mathrm{HCl}, \mathrm{pH}$ 7.5, $50 \mathrm{mM} \mathrm{NaCl}, 5 \mathrm{mM} \mathrm{MgCl} 2,1 \mathrm{mM} \mathrm{CaCl}_{2}, 0.2 \% \mathrm{NP}-40,1 \times$ Roche cOmplete EDTA-free). Chromatin was fragmented with $\mathrm{MNase}$ for $10 \mathrm{~min}$ at $37^{\circ} \mathrm{C}$ and digestion was stopped with $5 \mathrm{mM}$ EGTA at $65^{\circ} \mathrm{C}$ for $10 \mathrm{~min}$. The chromatin was resuspended in 1x NEBuffer 2.1 (NEB, \#B7202S) and dephosphorylated by addition of $5 \mu \mathrm{rSAP}$ (NEB, \#M0203) at $37^{\circ} \mathrm{C}$ for $45 \mathrm{~min} .5^{\prime}$ overhangs 
were generated with this pre-mix (50 mM NaCl, $10 \mathrm{mM}$ Tris, $10 \mathrm{mM} \mathrm{MgCl} 2,100 \mu \mathrm{g} / \mathrm{ml} \mathrm{BSA}, 2 \mathrm{mM}$ ATP, 3 mM DTT $8 \mu \mathrm{l}$ Large Klenow Fragment (NEB, \#M0210L) and $2 \mu \mathrm{l} \mathrm{T4} \mathrm{PNK} \mathrm{(NEB,} \mathrm{\# M0201L))}$ at $37^{\circ} \mathrm{C}$ for $15 \mathrm{~min}$. The DNA overhangs were filled with biotinylated nucleotides by addition of $100 \mu \mathrm{l}$ pre-mix (25 $\mu \mathrm{l} 0.4$ mM Biotin-dATP (Invitrogen, \#19524016), $25 \mu \mathrm{l} 0.4 \mathrm{mM}$ Biotin-dCTP (Invitrogen, \#19518018), 2 l 10 mM dGTP and 10 mM dTTP (stock solutions: NEB, \#N0446), 10 $\mu \mathrm{l}$ 10x T4 DNA Ligase Reaction Buffer (NEB \#B0202S), $0.5 \mu \mathrm{l}$ 200x BSA (NEB, \#B9000S), 38.5 $\mu \mathrm{H} 2 \mathrm{O}$ ) and incubation at $25^{\circ} \mathrm{C}$ for $45 \mathrm{~min}$. The reaction was stopped by addition of $12 \mu \mathrm{l} 0.5 \mathrm{M}$ EDTA (Invitrogen, \#15575038) at $65^{\circ} \mathrm{C}$ for $20 \mathrm{~min}$. After proximity ligation and removing unligated ends, the DNA was extracted with phenol/chloroform, purified with DNA clean \& Concentrator Kit (Zymo, \#4013), and the library was purified via 1.5\% agarose gel electrophoresis. Dynabeads ${ }^{\mathrm{TM}}$ MyOne ${ }^{\mathrm{TM}}$ Streptavidin C1 beads (Invitrogen, \#65001) were added to the sample and incubated at RT for $20 \mathrm{~min}$. The beads were washed twice and resuspended in $50 \mu \mathrm{l}$ TE buffer. Sequencing libraries were prepared with NEBNext® Ultra II DNA Library Prep Kit for Illumina $(\mathrm{NEB}, \# \mathrm{E} 7645)$ according to the manufacturer's protocol followed by PCR amplification, sample indexing, and DNA purification.

\section{Analysis of ATAC-seq data}

All libraries were sequenced using either Illumina Hiseq2500 v4 or Novaseq 6000 sequencers and $50 \mathrm{bp}$ paired-end format. Paired reads were aligned to the mouse reference genome $\mathrm{mm} 9$ using Bowtie2 (43). ATAC-seq reads were aligned using default parameters except $-\mathrm{X} 2000$-m 1. PCR duplicates were removed using Picard Tools (http://picard.sourceforge.net; https://broadinstitute.github.io/picard/). To adjust for fragment size, we aligned all reads as + strands offset by +4 bp and - strands offset by -5 bp (26). For all ATAC-seq datasets subnucleosome size and mono-nucleosome-size reads were separated by choosing fragments 50-115 bp and 180-247 bp in length, respectively. MACS2 (44) was used for peak calling of subnucleosomal reads, which represent bound transcription factors.

\section{ChIP-Seq data processing}

All reads were mapped to unique genomic regions using Bowtie2 (43) and the mm9 genome. PCR duplicates were removed using Picard Tools. MACS2 was used to call peaks using default parameters with IgG ChIP-seq data as a control.

\section{Transcription factor footprint analysis}

To analyze the footprints of TFs in ATAC-seq data, motifs on a set of peaks were used as anchors for running dnase_average_profile.py scripts of the Wellington program in ATAC-seq mode. The footprint $p$ values of all motifs on a set of peaks were derived using the wellington_footprints.py scripts of the Wellington (45) program in ATAC-Seq mode on read-normalized ATAC-seq subnucleosome-size (<115 bp) fragments.

\section{$\mathrm{Hi}-\mathrm{C}$ and Micro-C data processing}

Paired-end reads from $\mathrm{Hi}-\mathrm{C}$ experiments were aligned to the mouse $\mathrm{mm} 9$ reference genome using Juicer (46). After PCR duplicates and low-quality reads were removed, high-quality reads were assigned to Dpnll restriction fragments, and $\mathrm{Hi}-\mathrm{C}$ interaction contacts where mapped in a binned matrix to create a hic file. SIP was used to call CTCF loops in the Hi-C interaction matrix (27). All statistically significant peaks were post-filtered for observed values greater than $12 \mathrm{Hi}-\mathrm{C}$ contacts, observed over expected values greater than $2 \mathrm{Hi}-\mathrm{C}$ contacts, and interactions that are less than $5 \mathrm{Mb}$ apart. The WashU Epigenome browser was used to obtain arc views of significant loops. FitHi-C (47) was used to call significant interaction at $25 \mathrm{~kb}$ resolution from the second pass with a $q$-value threshold of $q>0.001$.

\section{Supplemental Figures and Tables}


A

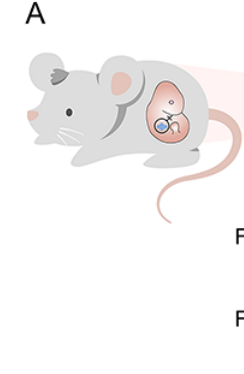

Transgenerational F3

D

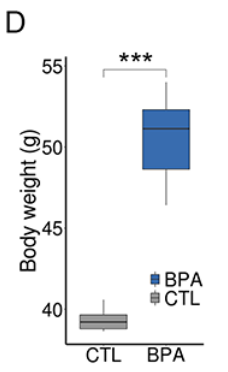

Pregnant females exposed to BPA

F0

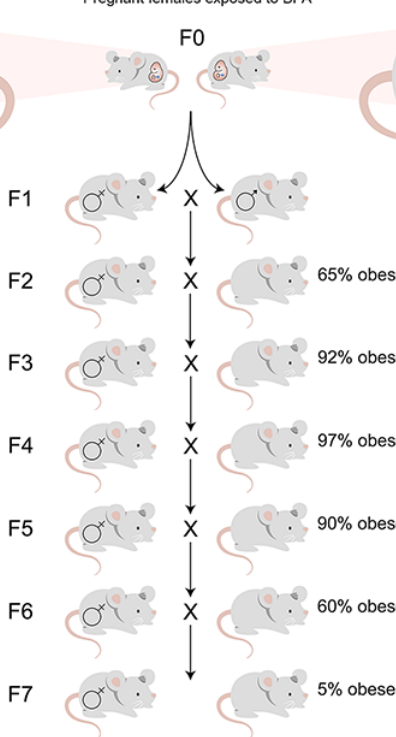

B

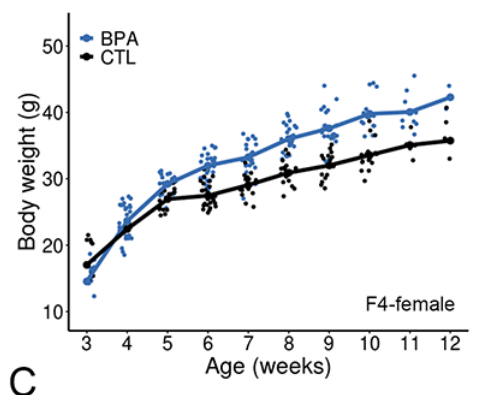

C

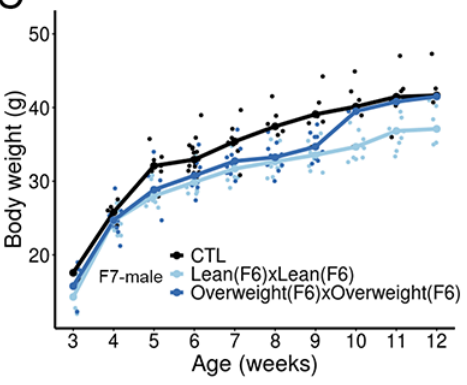

F DAPI Sca-1 Merged
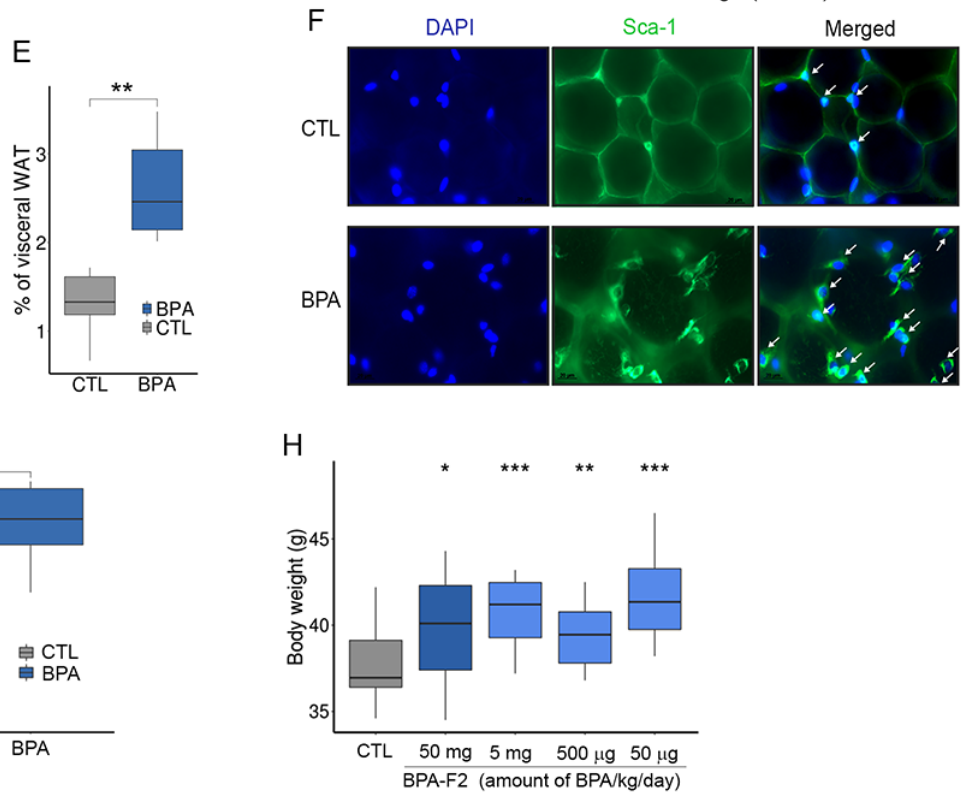

Fig S1. Outline of crosses used to obtain different generations of BPA-exposed mice.

(A) Two different pregnant CD1 females (F0) were injected with BPA during days E7.5-E13.5 of embryonic development when the germline of the embryo (labeled in blue) is undergoing demethylation. Male or female F1 progeny from each F0 female are crossed to obtain the F2 generation, which arises from the BPA-exposed germline. F2 males and females are crossed to obtain F3, which is the first generation whose cells were not directly exposed to BPA. The percentage of obese mice in each generation is indicated. (B) Changes in body weight with time for BPA-F4 females. (C) Changes in body weight with time for control and F7 males arising from crosses between BPA-F6 lean or BPA-F6 overweight animals. (D) Average body weight for BPAF4 males. (E) Average percentage of visceral fat with respect to body weight for BPA-F4 males. (F) Immunofluorescence microscopy of visceral fat tissue from control and BPA-F4 males stain with antibodies to Sca-1 to visualize adipocyte progenitor cells. (G) Percentage of adipocyte progenitor cells in visceral fat tissue of control and BPA-F4 males. (H) Average body weight of BPA-F2 males progeny from an F0 female exposed to different amounts of BPA as indicated. 

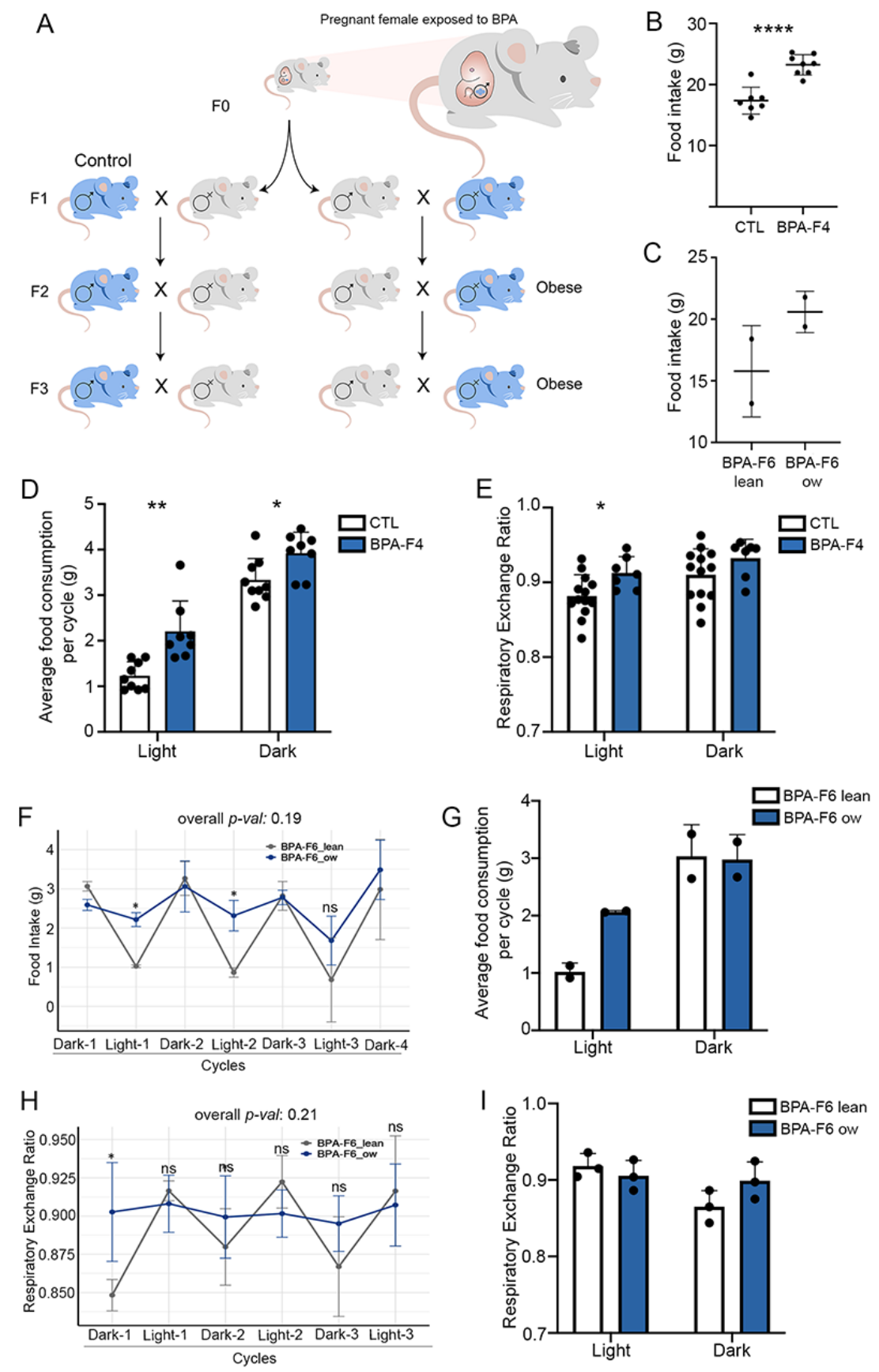

Fig. S2. Transgenerational effects of BPA on body weight.

(A) Outcrosses between BPA-F1 males or females and control mice of the opposite sex. (B) Differences in total daily food intake between control and BPA-F4 males. (C) Differences in total daily food intake between overweight and lean BPA-F6 males (D) Average food consumption during the light and dark light cycles in control and BPA-F4 males. (E) Respiratory exchange ratio during the light and dark light cycles in control and BPA-F4 males. (F) Food consumption during the light and dark light cycles in control and BPA-F6 overweight and lean males. (G) Average food consumption per light or dark light cycles for BPA-F6 overweight and lean males. (H) Respiratory exchange ratio during the light and dark light cycles in BPA-F6 overweight and lean males. (I) Average respiratory exchange ratio during the light and dark light cycles in BPAF6 overweight and lean males. 

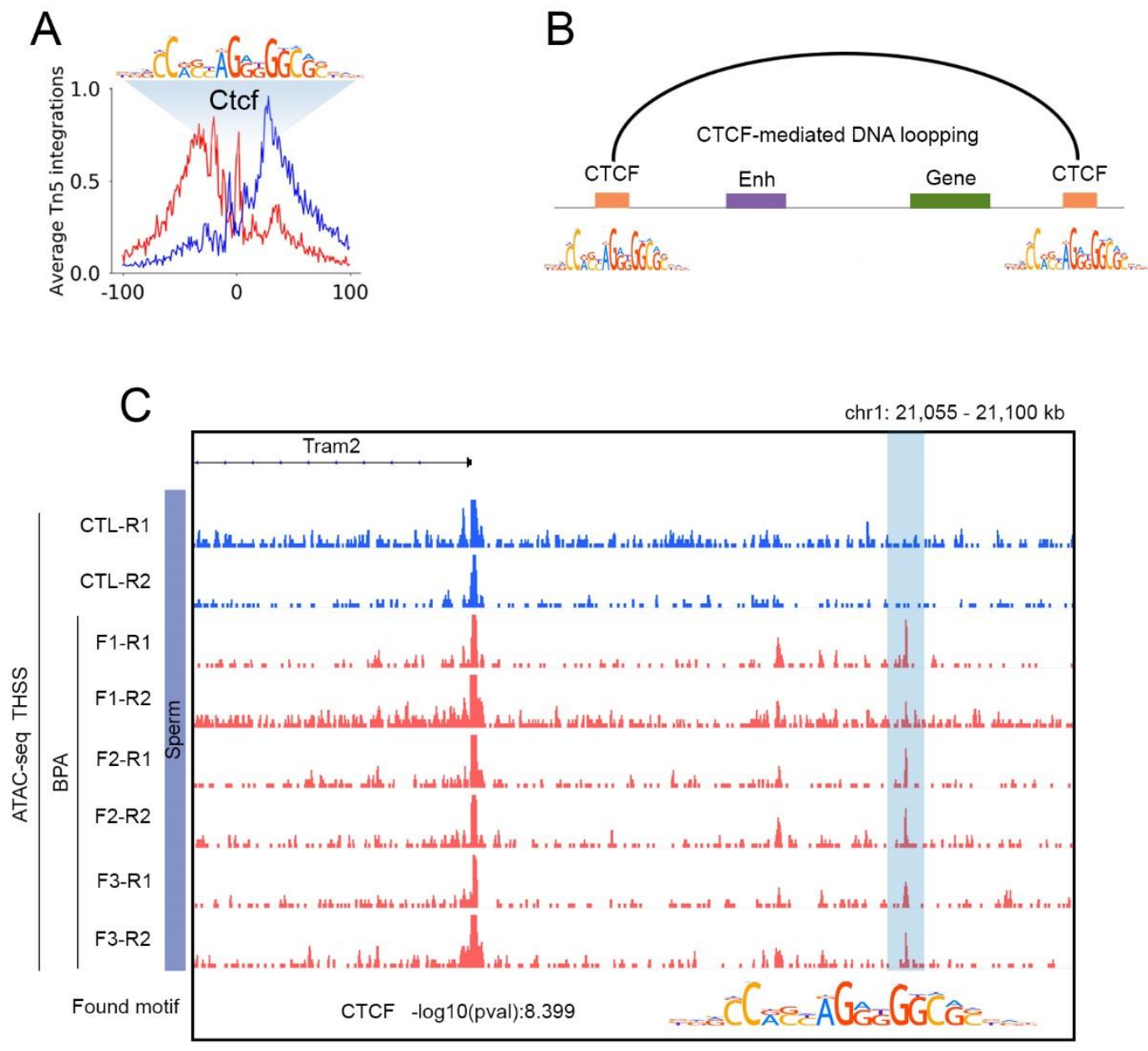

Fig. S3. BPA exposure results in new CTCF sites in sperm.

(A) ATAC-seq sites containing the CTCF motif show a footprint similar to that previously shown to be present at CTCF-bound sites in the genome. (B) Analysis of Hi-C data suggest that new CTCF sites form loops with other CTCF sites in the genome. (C) Example of a new CTCF site present in the 5' region of the Tram2 gene in sperm of BPA-F1-3 but not in control. 

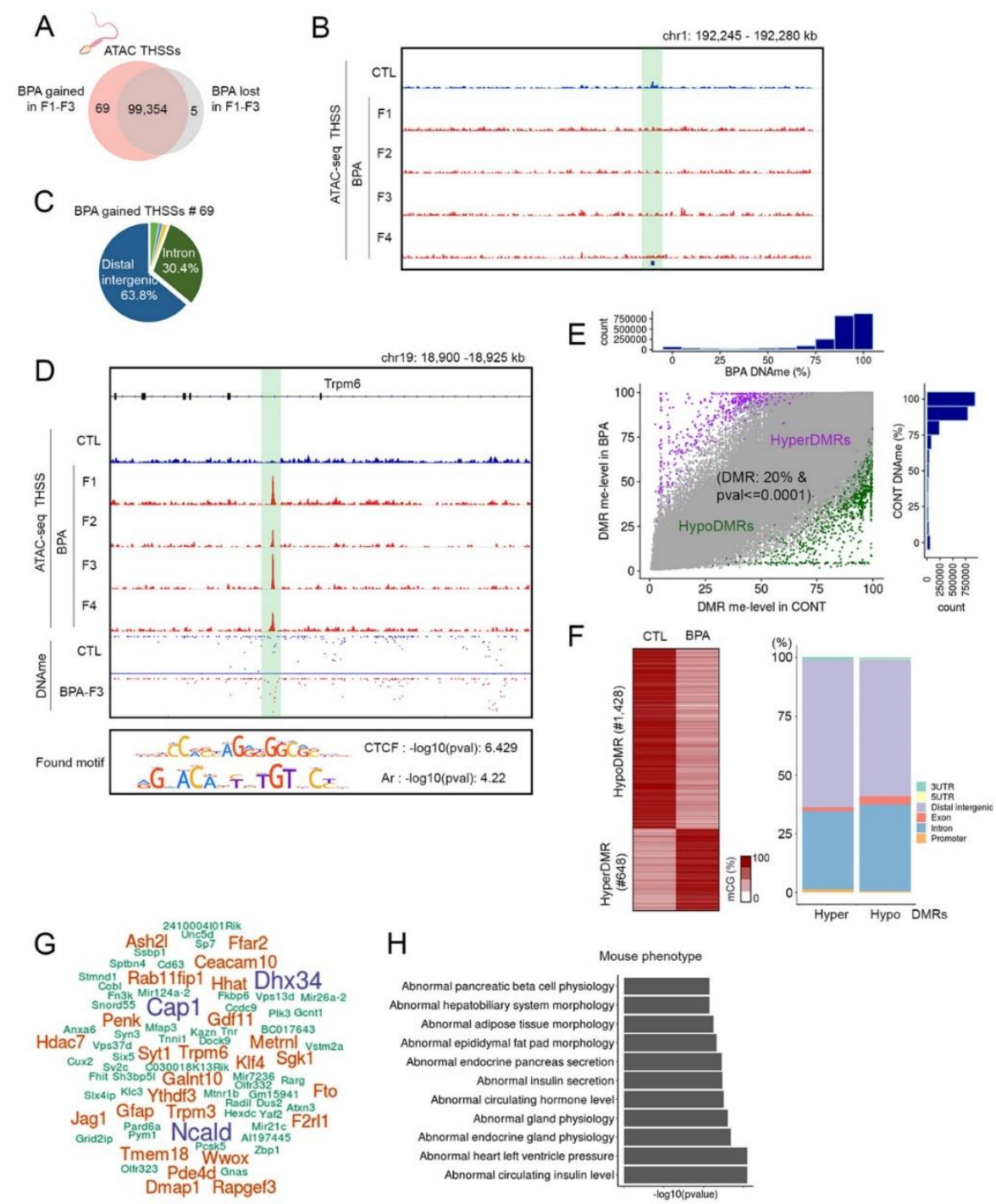

Fig. S4. New ATAC-seq sites in BPA-Fi are differentially methylated.

(A) Overlap between ATAC-seq peaks in sperm for BPAF1-F3 and controls showing the peaks gained and lost in the progeny of BPA-exposed females. (B) An example of an ATAC-seq peak present in control but not in BPA-F1-F3. (C, Distribution of ATAC-seq peaks present in BPA-F1F3 but not in controls with respect to gene features. (D) An example of an ATAC-seq peak present in BPA-F1-F3 but not in controls in the Trpm6 gene. The summit of the peak contains motifs for CTCF and Ar. The site is hypomethylated in BPA-F3 with respect to control. (E) Analysis of GWBS-seq data showing differentially methylated regions (DMRs) between BPA-F3 and control sperm. (F) Heatmap showing different in DNA methylation levels at regions hyper- and hypomethylated in BPA-F3 sperm with respect to control (left). DMRs are enriched at intergenic and intronic regions, suggesting they may correspond to enhancer sequences (right). (G) Genes contacted by the 69 ATAC-seq accessible sites present in BPA-F1-F4 whose RNA levels are higher in sperm from BPA-F3 mice than control. Green represents a 2-fold or more increase in expression; orange represents genes shown to be involved in obesity in humans based on results from GWAS studies; purple represents genes involved in obesity and with increased levels in sperm. (H) Phenotypes of mice mutant in genes contacted by the 69 ATAC-seq sites present in BPA-F1-F4 but not in controls. 

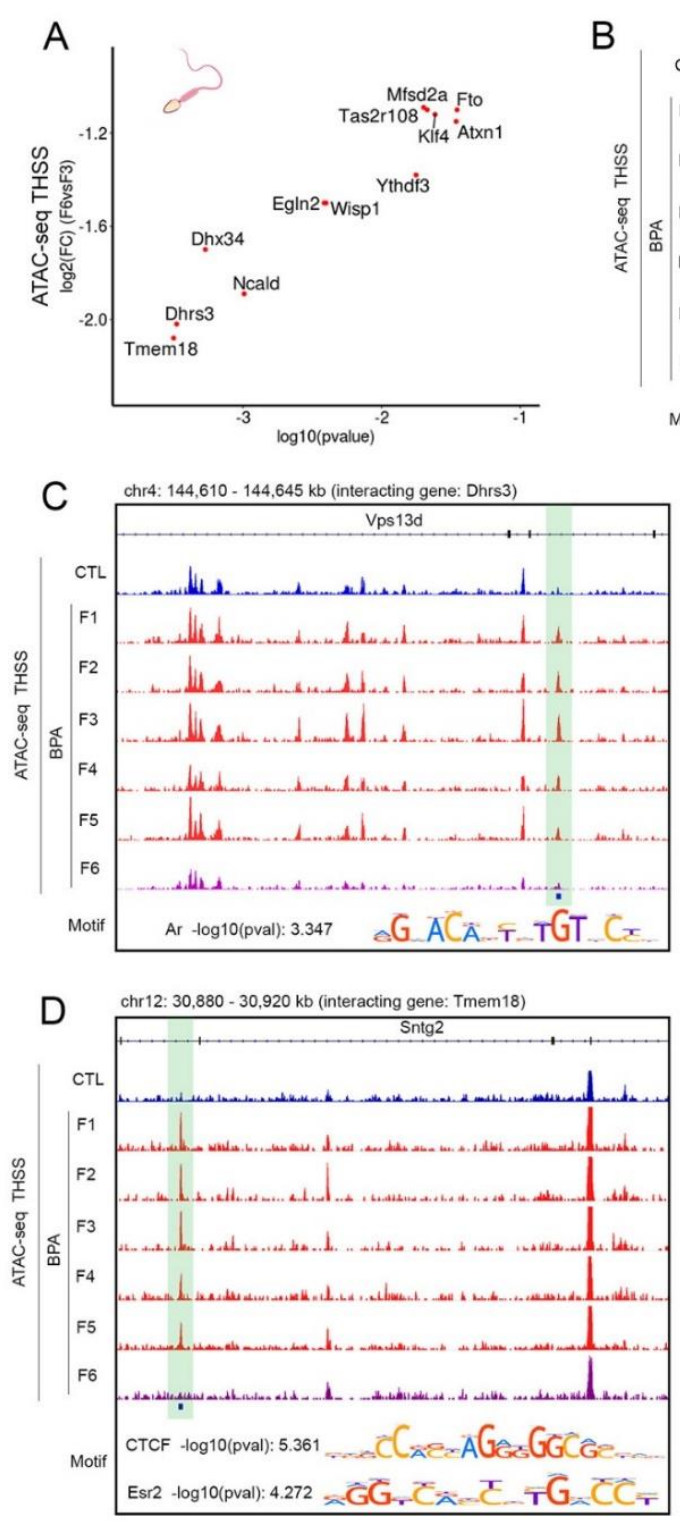
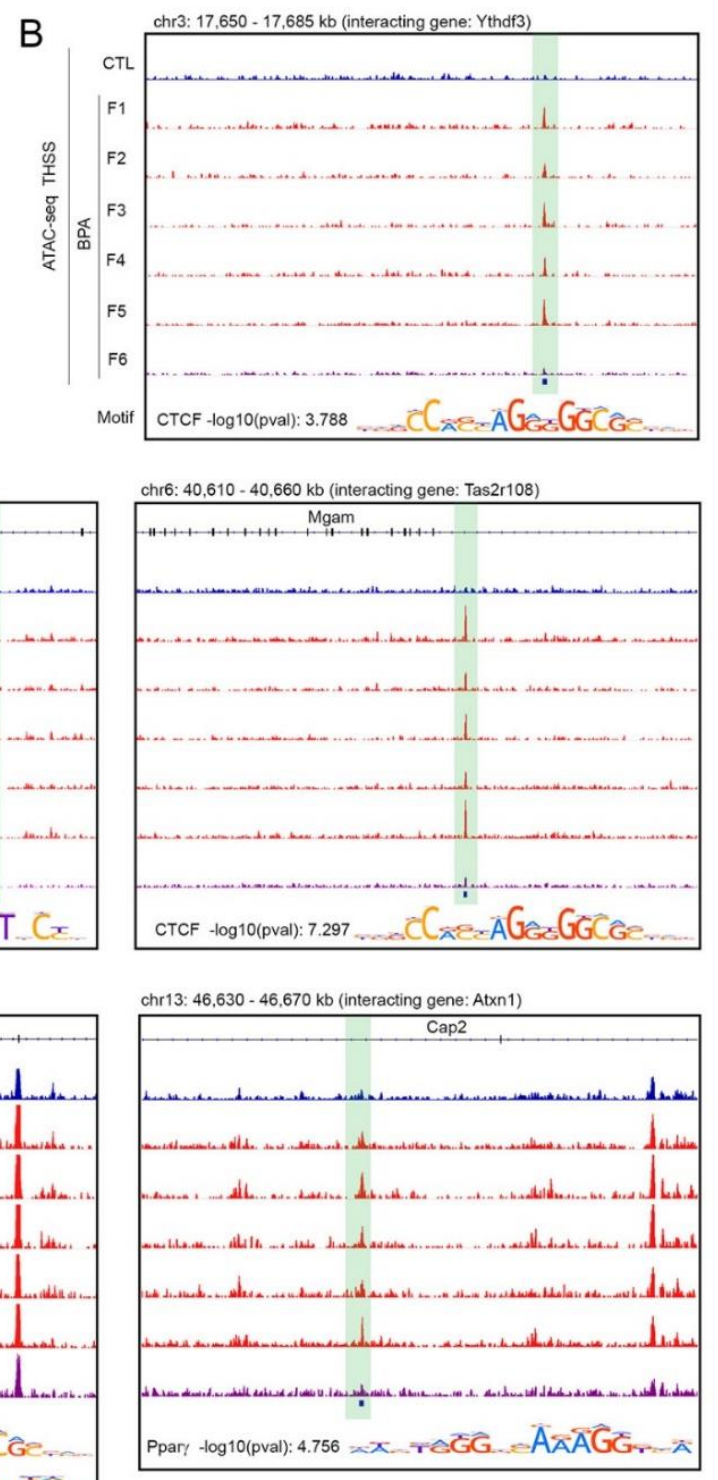

Fig S5. Examples of ATAC-seq sites present in BPA-F1-F5 but absent in BPA-F6.

(A) Genes whose promoters are contacted with the highest frequency by the 12 ATAC-seq sites present in BPA-F1-F5 but not $\mathrm{F} 6$ based on significant interactions determined by Fit-Hi-C using $\mathrm{Hi}-\mathrm{C}$ data obtained in sperm of BPA-F3 and CTL-F3 mice. (B) Example of one of the 12 ATACseq sites present in an intergenic region; this site contains a CTCF motif and it preferentially contacts the promoter of the Ythdf3 gene. (C) examples of 2 of the 12 ATAC-seq sites present in gene introns. These sites contain binding motifs for Ar and CTCF, respectively. Rather than interacting preferentially with the promoters of the genes in which they are located, the site located in an intron of Vps13d interacts with the Dhrs3 gene (left panel), whereas the enhancer located in an intron of Mgam preferentially interacts with the promoter of Tas2r108 (right panel). (D) additional examples of 2 of the 12 ATAC-seq sites present in gene introns. These sites contain binding motifs for CTCF and Esr2, and Ppary, respectively. Rather than interacting preferentially with the promoters of the genes in which they are located, the site located in an intron of Sntg2 interacts with the Tmem18 gene (left panel), whereas the enhancer located in an intron of Cap2 preferentially interacts with the promoter of Atxn1 (right panel). 

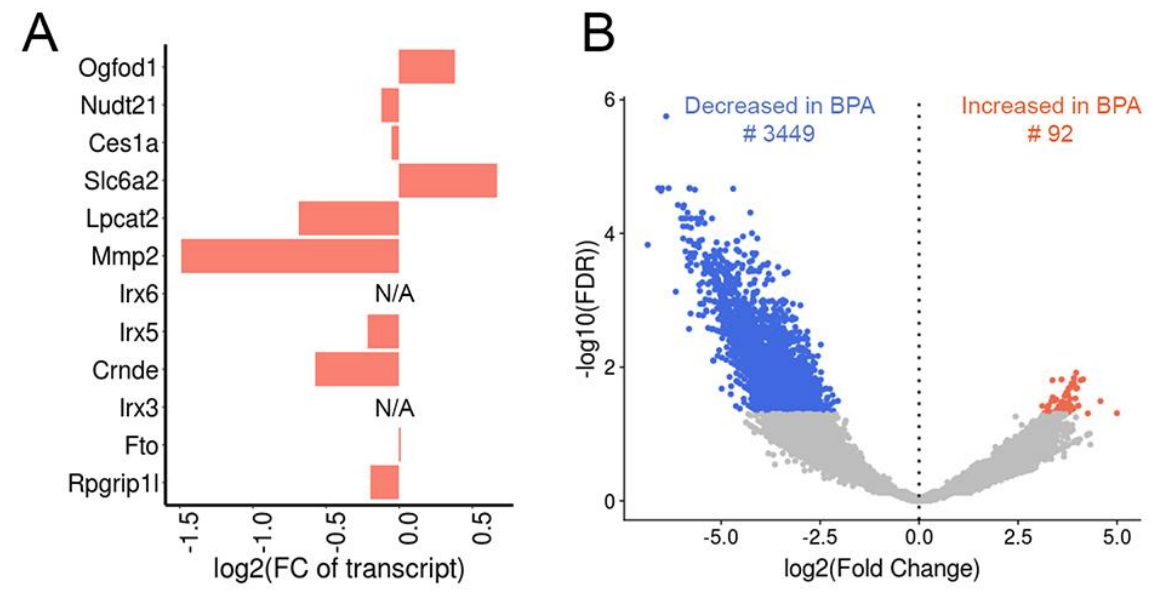

C
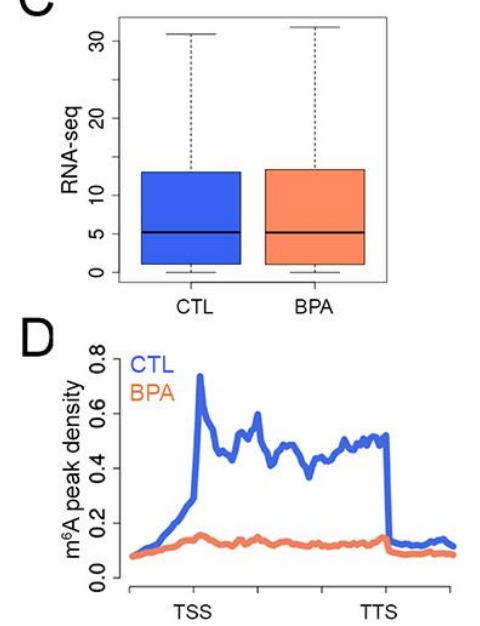
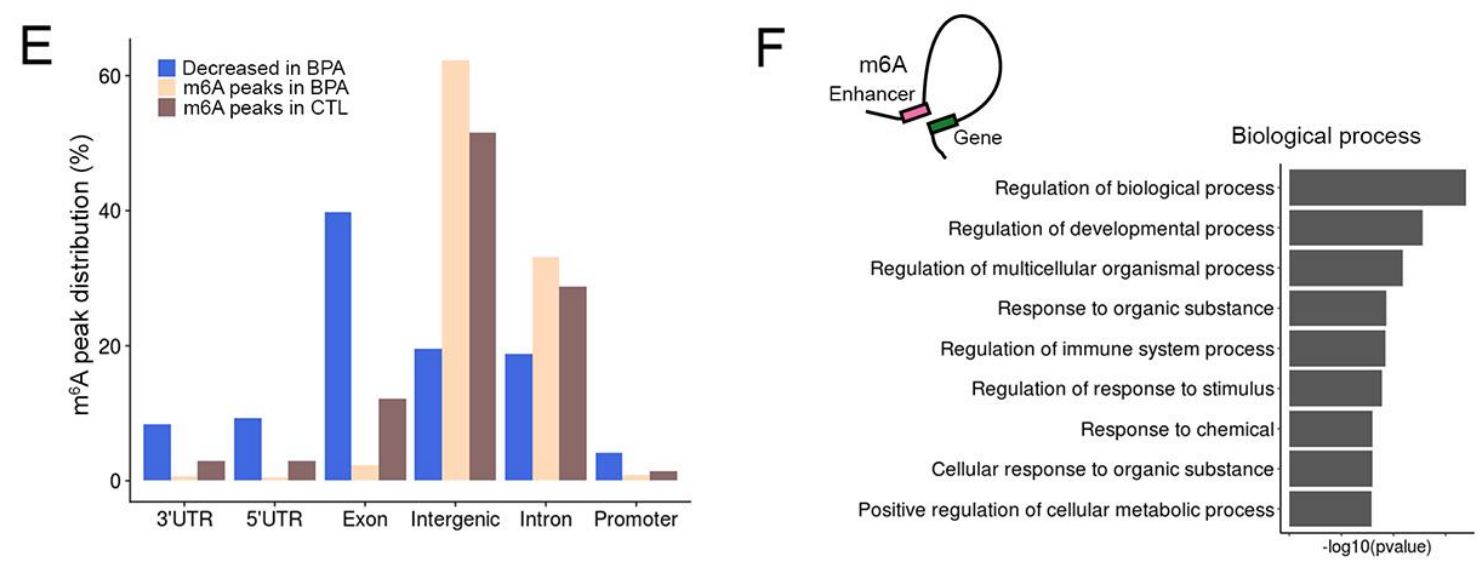

Fig S6. Effect of BPA-induced epigenetic alterations in Fto on $\mathrm{m}^{6} \mathrm{~A}$ levels in sperm RNAs. (A) relative RNA levels in sperm of BAP-F3 and control transcribed from genes present in the region surrounding Fto. (B) RNAs whose $\mathrm{m}^{6} \mathrm{~A}$ levels are altered in BPA-F3 sperm with respect to control. (C) RNA levels are not different between sperm of BPA-F3 and control mice. (D) Levels of $\mathrm{m6A}$ at sites located in mRNAs are lower in sperm from BPA-F3 mice with respect to control. (E) location of sites in RNAs modified by $\mathrm{m}^{6} \mathrm{~A}$ with respect to genomic features. (F) Biological processes in which a specific set of genes are involved; the promoters of these genes are contacted by enhancers whose eRNAs show decreased levels of $m^{6} A$ in BPA-F3 with respect to control. 

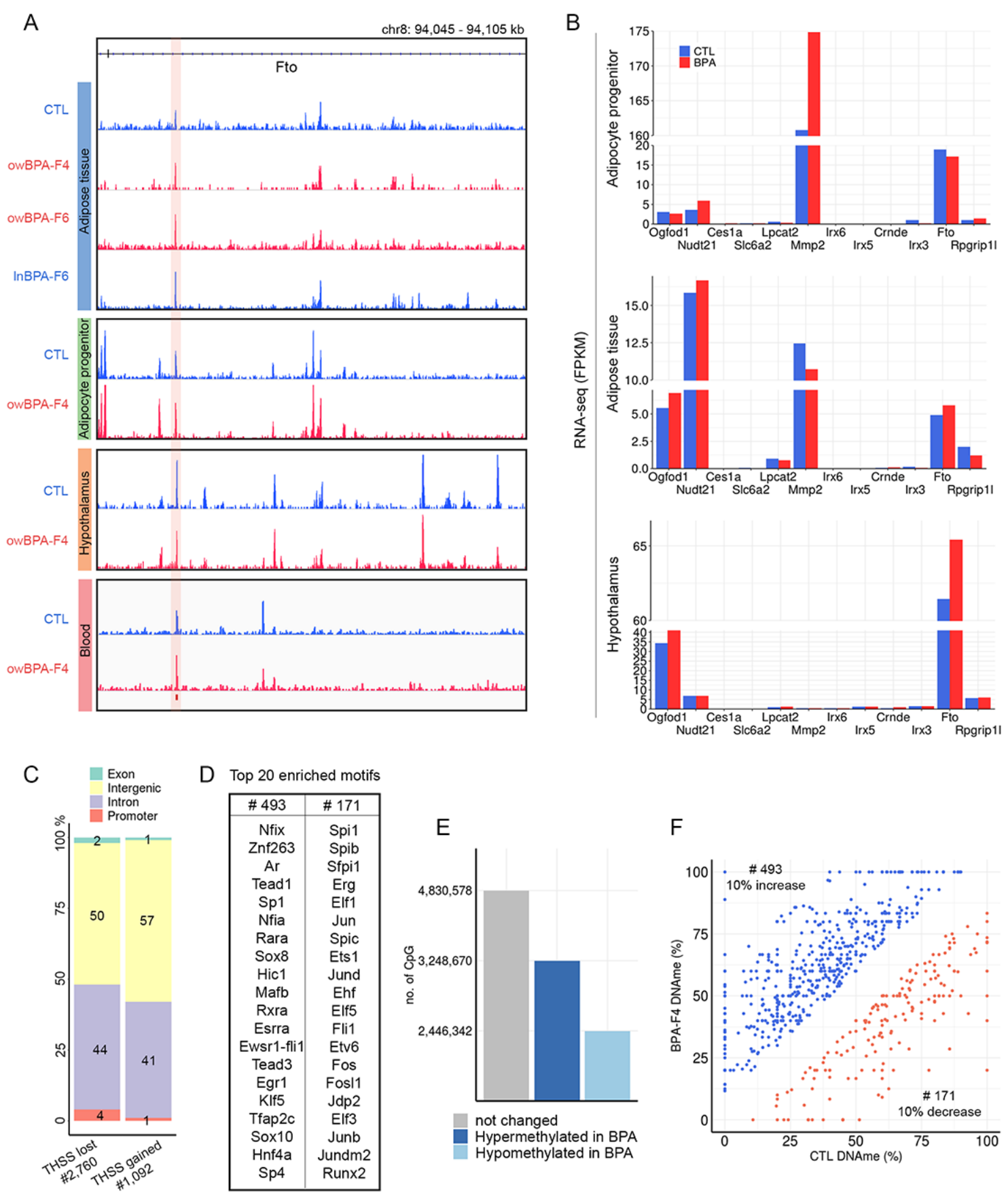

Fig. S7. Changes in ATAC-seq and DNA methylation in adult tissues of control and BPAF4 mice

(A) Genome browser view of the last intron of the Fto gene showing ATAC-seq peaks in various adult tissues of control and BPA-F4 males. (B) Gene expression from RNA-seq in adult tissues from control and BPA-F4 males. (C) Distribution of differential ATAC-seq peaks from subnucleosome size reads (THSSs) from visceral fat tissue of control and BPA-F4 males. (D) Top 20 enriched TF binding motifs in differential ATAC-seq peaks with differential DNA methylation levels (see panel F) from visceral adipose tissue of control and BPA-F4 males. (E) Changes in DNA methylation from GWBS data obtained from visceral fat tissue of control and BPA-F4 males. (F). Changes in DNA methylation in visceral fat tissue between control and BPA-F4 males at differential ATAC-seq peaks. 


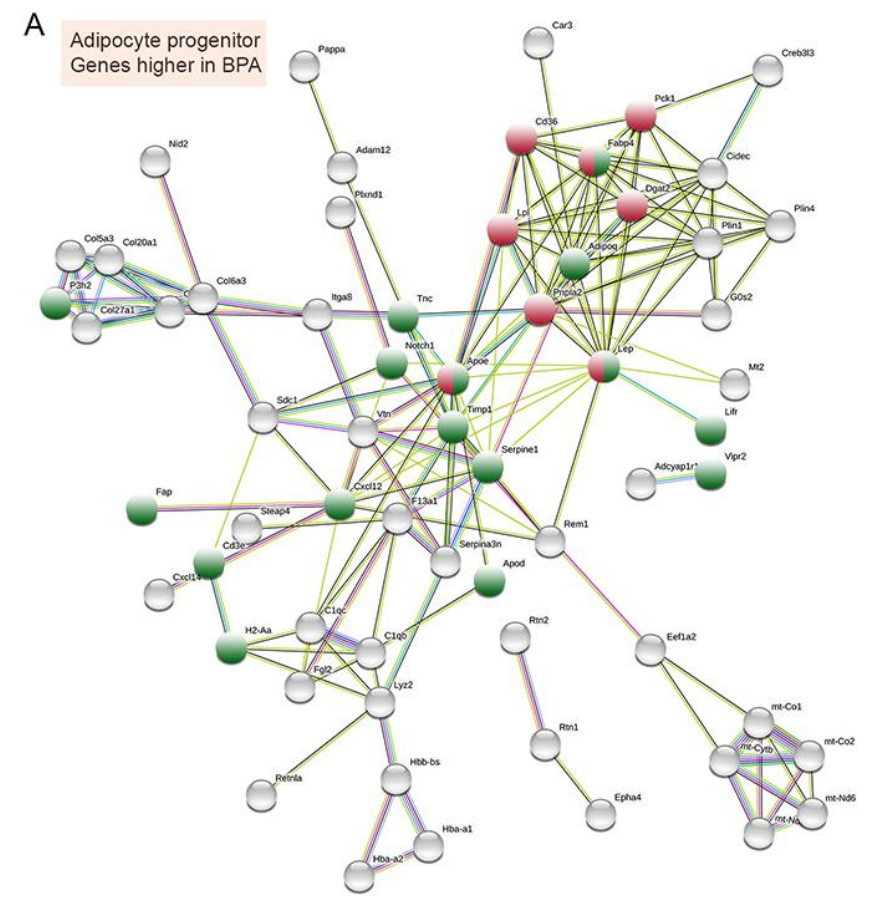

B

\begin{tabular}{|c|c|c|}
\hline Biological Process (Gene Ontology) & FDR & \\
\hline Regulation of cell population proliferation & 0.002 & 0 \\
\hline $\begin{array}{l}\text { Gycerol metabolic process } \\
\text { Triglyceride metabolic process } \\
\text { Lipid homeostasis }\end{array}$ & $\begin{array}{l}0.0042 \\
0.00026 \\
0.002\end{array}$ & 0 \\
\hline Biological Process (Gene Ontology) & FDR & \\
\hline $\begin{array}{l}\text { Regulation of cell morphogenesis in } \\
\text { differentiation }\end{array}$ & 0.0168 & 0 \\
\hline $\begin{array}{l}\text { Regulation of cellular protein metabolic } \\
\text { process }\end{array}$ & 0.0234 & 0 \\
\hline
\end{tabular}

C

Adipose tissue Genes higher in BPA
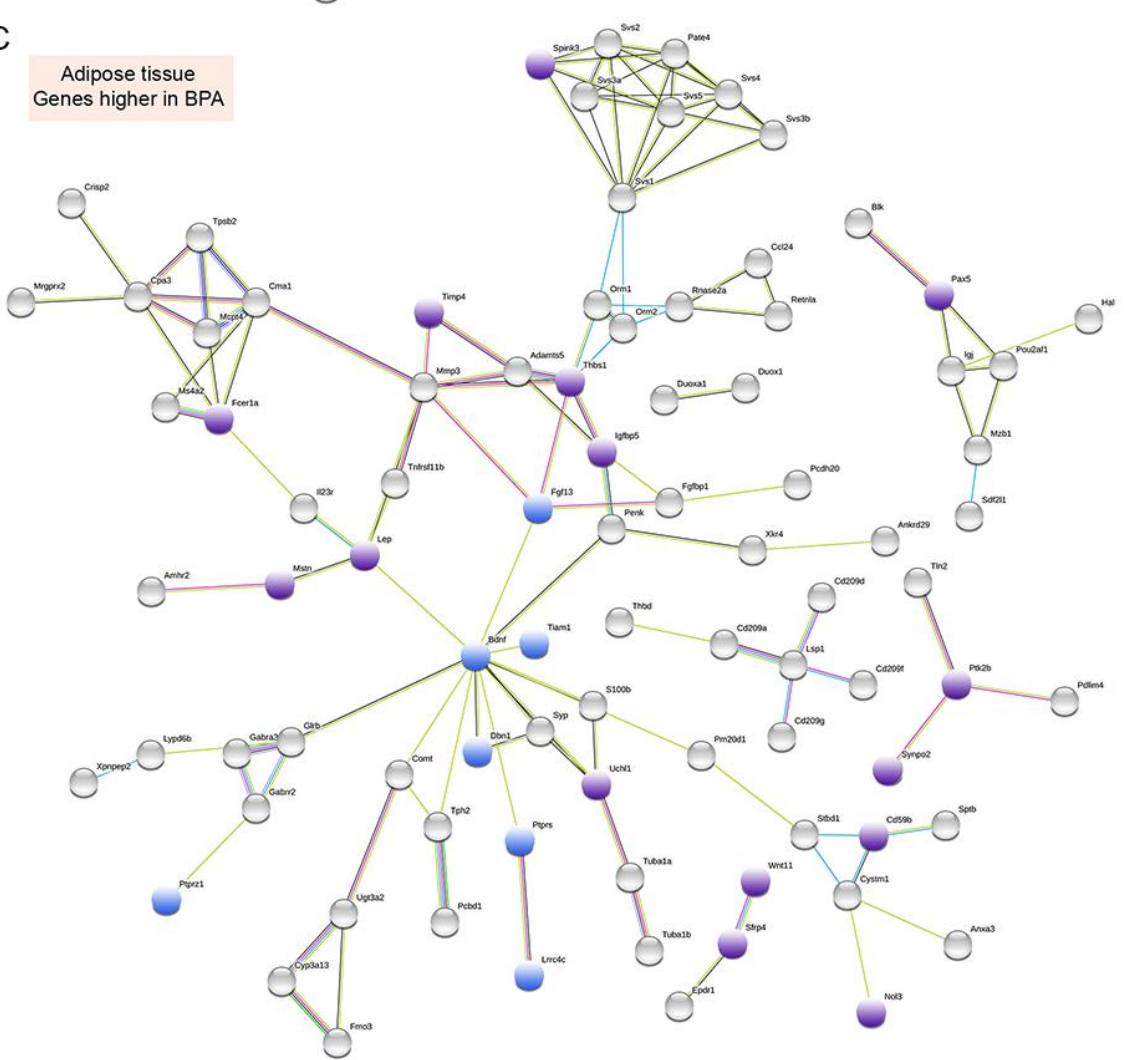

Fig. S8 Functional relationships between genes with altered expression in fat tissue

(A) Functional interactions between genes differentially expressed in adipocyte progenitor cells from control and BPA-F4 males. (B) GO analysis of genes differentially expressed in adipocyte progenitor cells from control and BPA-F4 males. (C) Functional interactions between genes differentially expressed in visceral fat tissue from control and BPA-F4 males. (D) GO analysis of genes differentially expressed in visceral fat tissue from control and BPA-F4 males. 
bioRxiv preprint doi: https://doi.org/10.1101/2020.11.20.391672; this version posted November 20, 2020. The copyright holder for this preprint (which was not certified by peer review) is the author/funder, who has granted bioRxiv a license to display the preprint in perpetuity. It is made available under aCC-BY-NC-ND 4.0 International license.

\section{Supplementary Tables}

Supplementary Tables 1 and 2 are provided as Excel files. 